TRANSACTIONS OF THE

AMERICAN MATHEMATICAL SOCIETY

Volume 365, Number 6, June 2013, Pages 3227-3253

S 0002-9947(2012)05735-7

Article electronically published on September 19, 2012

\title{
ZERO-FREE REGIONS FOR DIRICHLET SERIES
}

\author{
C. DELAUNAY, E. FRICAIN, E. MOSAKI, AND O. ROBERT
}

\begin{abstract}
In this paper, we are interested in explicit zero-free discs for some Dirichlet series and we also study a general Beurling-Nyman criterion for $L$ functions. Our results generalize and improve previous results obtained by N. Nikolski and by A. de Roton. As a concrete application, we get, for example, a Beurling-Nyman type criterion for the Siegel zero problem.
\end{abstract}

\section{INTRODUCTION}

In this article, we are interested in zero-free regions for functions that are obtained as a meromorphic continuation of Dirichlet series $s \mapsto \sum_{n>1} a_{n} n^{-s}$. Such a study arises naturally in various fields of mathematics such as functional analysis and number theory.

The particular case of the Riemann zeta function has been most studied, and is related to the Riemann Hypothesis, asserting that the zeta function does not vanish on the half-plane $\Re(s)>1 / 2$. Several attempts have been made in the direction of solving or reformulating this conjecture.

In his thesis, B. Nyman Nym50 gave a reformulation of the Riemann Hypothesis by means of functional analysis. More precisely let $\{\cdot\}$ denote the fractional part and let $\mathcal{N}$ be the set of functions

$$
f(x)=\sum_{j=1}^{n} c_{j}\left\{\frac{\theta_{j}}{x}\right\},
$$

where $0<\theta_{j} \leq 1, c_{j} \in \mathbb{C}$ and

$$
\sum_{j=1}^{n} c_{j} \theta_{j}=0 .
$$

Then Nyman proved that the Riemann Hypothesis holds if and only if the characteristic function $\chi_{(0,1)}$ of the interval $(0,1)$ belongs to the closure of $\mathcal{N}$ in $L^{2}(0,1)$. Later on, A. Beurling Beu55] extended this result by proving that if $1<p<+\infty$, then the Riemann zeta function has no zero in $\Re(s)>1 / p$ if and only if $\chi_{(0,1)}$ belongs to the closure of $\mathcal{N}$ in $L^{p}(0,1)$. The case $p=1$ has been investigated in [BF84]. After the works of Beurling and Nyman, several results occur in this direction; see for example Nik95, BS98, BDBLS00, Bur02, BD03, and dR07a].

Received by the editors January 6, 2011 and, in revised form, September 21, 2011 and October $28,2011$.

2010 Mathematics Subject Classification. Primary 11M26, 30H10.

Key words and phrases. Dirichlet series, Beurling-Nyman criterion, Hardy spaces, zeros of $L$-functions.

This work was supported by the ANR project no. 07-BLAN-0248 "ALGOL", the ANR project no. 09-BLAN-005801 "FRAB" and the ANR project no. 08-BLAN-0257 "PEPR"..

(C)2012 American Mathematical Society 
In particular, N. Nikolski Nik95 gave, in a way, an effective version of the Beurling-Nyman criterion and produced explicit zero-free regions for the Riemann zeta function: let $r>0$ and $\lambda \in \mathbb{C}$ with $\Re(\lambda)>0$ be fixed parameters and let $\tilde{K}_{r}$ be the subspace of $L^{2}((0,1), d x / x)$ spanned by the functions

$$
E_{\alpha, r}(x)=x^{r}\left(\left\{\frac{\alpha}{x}\right\}-\alpha\left\{\frac{1}{x}\right\}\right), \quad 0<x<1,
$$

where $0 \leq \alpha \leq 1$. Then the zero-free regions obtained by Nikolski are domains of the form

$$
r+\left\{\mu \in \mathbb{C}:\left|\frac{\mu-\lambda}{\mu+\bar{\lambda}}\right|^{2}<1-2 \Re(\lambda) \tilde{d}_{r}(\lambda)^{2}\right\},
$$

where $\tilde{d}_{r}(\lambda)=\operatorname{dist}\left(x^{\lambda}, \tilde{K}_{r}\right)$ is the distance in $L^{2}((0,1), d x / x)$ between $x^{\lambda}$ and the subspace $\tilde{K}_{r}$. In the case $\lambda=r=1 / 2$, if $\tilde{d}_{r}(\lambda)=0$ the region (1.2) corresponds to the half-plane $\Re(\mu)>1 / 2$ and Nikolski recovers Nyman's result (note that in this case, $\tilde{d}_{1 / 2}(1 / 2)=0$ if and only if $\chi_{(0,1)}$ belongs to the closure of $\mathcal{N}$ in $L^{2}(0,1)$; see section 7.1.1).

More recently, A. de Roton dR07a generalized Nyman's work to the Selberg class of Dirichlet functions and reformulated the Generalized Riemann Hypothesis. Finally let us mention that in BDBLS00 and Bur02 interesting lower bounds are obtained. First the authors proved in BDBLS00] that if $0<\lambda \leq 1$ and $\mathcal{N}_{\lambda}^{\sharp}$ is the closed span in $L^{2}(0,+\infty)$ of functions

$$
f(x)=\sum_{j=1}^{n} c_{j}\left\{\frac{\theta_{j}}{x}\right\}, \quad x>0,
$$

where $\lambda<\theta_{j} \leq 1, c_{j} \in \mathbb{C}$, then the Riemann Hypothesis holds if and only if $\lim _{\lambda \rightarrow 0} d(\lambda)=0$, where $d(\lambda)=\operatorname{dist}\left(\chi_{(0,1)}, \mathcal{N}_{\lambda}^{\sharp}\right)$. In other words, we can drop the condition (1.1) in the Nyman theorem. Furthermore they proved that

$$
\liminf _{\lambda \rightarrow 0} d(\lambda) \sqrt{\log \frac{1}{|\lambda|}}>0 .
$$

Then this lower bound was improved by J.-F. Burnol in Bur02 and generalized by de Roton in [R06] for the Selberg class.

The aim of our article is to make a further investigation of Nikolski's work in a more general situation including, in particular, the Selberg class. Associated to some auxiliary function $\varphi:[0, \infty) \rightarrow \mathbb{C}$, we introduce a class of spaces $K_{r}$ (in particular, in the case of the Riemann zeta function, $K_{r}$ coincides with $\tilde{K}_{r}$ for a special choice of $\varphi$ ). Then, for a large class of Dirichlet series, we obtain explicit zero-free regions that are larger than Nikolski's regions in the case of zeta. For some well-chosen parameters, if $\operatorname{dist}\left(x^{\lambda}, K_{r}\right)=0$ our zero-free regions correspond to $\Re(\mu)>r$ and we recover (and in fact improve a little bit) the results of de Roton concerning the reformulation of the Generalized Riemann Hypothesis. Finally, we investigate several explicit applications. In particular, we give zero-free regions for the zeta function and Dirichlet $L$-functions; it should be pointed out that the domains obtained have the property of being explicit, but they do not have the ambition of competing with the classical nonzero regions that are involved, for instance, in the prime number theorem. Another application we obtain is somehow 
a Beurling-Nyman criterion for the Siegel zero problem of Dirichlet $L$-functions. As far as we know, it seems to be a new criterion concerning this question.

\section{Notation And statements of the main Results}

For a generic $s \in \mathbb{C}$, we denote by $\sigma$ (respectively $t$ ) its real (respectively imaginary) part so that we have $s=\sigma+i t$. For $s \in \mathbb{C}$, we denote by $\Pi_{s}$ the half-plane defined by

$$
\Pi_{s}=\Pi_{\sigma}=\{z \in \mathbb{C}: \Re(z)>\sigma\} .
$$

For the sequel, we fix a Dirichlet series

$$
L(s)=\sum_{n \geq 1} \frac{a_{n}}{n^{s}}
$$

satisfying the following conditions:

- For every $\varepsilon>0$, we have $a_{n} \ll_{\varepsilon} n^{\varepsilon}$.

- There exists $\sigma_{0}<1$ such that the function $s \mapsto L(s)$ admits a meromorphic continuation to $\Re(s)>\sigma_{0}$ with a unique pole of order $m_{L}$ at $s=1$.

- The function $s \mapsto(s-1)^{m_{L}} L(s)$ is analytic with finite order in $\Pi_{\sigma_{0}}$.

The growth condition on the coefficients $\left(a_{n}\right)_{n}$ implies that $L(s)$ is an absolutely convergent Dirichlet series for $\Re(s)>1$. The second condition tells us that the function $(s-1)^{m_{L}} L(s)$ can be analytically continued in some half-plane $\Pi_{\sigma_{0}}$ which contains $\Pi_{1}$. Note that we do not require either an Euler product or a functional equation for $L(s)$.

We also consider an auxiliary function $\varphi:[0,+\infty[\longrightarrow \mathbb{C}$ satisfying the following conditions:

- $\varphi$ is supported on $[0,1]$.

- $\varphi$ is locally bounded on $(0,1)$.

- $\varphi(x)=O\left(x^{-\sigma_{0}}\right)$ when $x \rightarrow 0$.

- $\varphi(x)=O\left((1-x)^{-\sigma_{1}}\right)$ when $x \rightarrow 1^{-}$, for some $\sigma_{1}<1 / 2$.

The fact that $\sigma_{1}<1$ is sufficient for the integral

$$
\int_{0}^{1} \varphi(t) t^{s} \frac{d t}{t}
$$

to be absolutely convergent for $\Re(s)>\sigma_{0}$. Hence, the Mellin transform $\widehat{\varphi}$ of $\varphi$ is analytic on $\Pi_{\sigma_{0}}$. The condition $\sigma_{1}<1 / 2$ will become clearer in Lemma 3.3. Recall that the (unnormalized) Mellin transform of a Lebesgue-measurable function $\varphi:[0,+\infty[\rightarrow \mathbb{C}$ is the function $\widehat{\varphi}$ defined by

$$
\widehat{\varphi}(s)=\int_{0}^{+\infty} \varphi(t) t^{s} \frac{d t}{t} \quad(s \in \mathbb{C}),
$$

whenever the integral is absolutely convergent. Let $H^{2}\left(\Pi_{\sigma}\right)$ be the Hardy space of analytic functions $f: \Pi_{\sigma} \rightarrow \mathbb{C}$ such that $\|f\|_{2}<\infty$, where

$$
\|f\|_{2}=\sup _{x>\sigma}\left(\int_{-\infty}^{+\infty}|f(x+i t)|^{2} d t\right)^{\frac{1}{2}} .
$$

Then the (normalized) Mellin transform

$$
\begin{array}{cc}
\mathcal{M}: \quad L_{*}^{2}\left((0,1), \frac{d t}{t^{1-2 \sigma}}\right) & \longrightarrow H^{2}\left(\Pi_{\sigma}\right) \\
\varphi & \longmapsto \frac{1}{\sqrt{2 \pi}} \widehat{\varphi}
\end{array}
$$


is a unitary operator (use the Paley-Wiener theorem and the change of variables going from the Fourier transform to the Mellin transform; for instance, see Nik02, p. 166]). Here, we write $L_{*}^{2}\left((0,1), \frac{d t}{t^{1-2 \sigma}}\right)$ for the subspace of functions in $L^{2}\left((0,+\infty), \frac{d t}{t^{1-2 \sigma}}\right)$ that vanish almost everywhere on $(1,+\infty)$.

We define

$$
\psi(u)=\operatorname{res}\left(L(s) \hat{\varphi}(s) u^{s}, s=1\right)-\sum_{n<u} a_{n} \varphi\left(\frac{n}{u}\right) \quad\left(u \in \mathbb{R}_{+}\right),
$$

where $\operatorname{res}(F(s), s=1)$ denotes the residue of the meromorphic function $F$ at $s=1$. The method that is going to be explored depends on the fact that the function $\psi$ belongs to $L^{2}\left((1,+\infty), \frac{d u}{u^{1+2 r}}\right)$ for a certain real number $r>\sigma_{0}$. The following gives a criterion for this property.

Theorem 2.1. Let $r>\sigma_{0}$. If $m_{L} \geq 1$, we assume furthermore that $r \neq 1$. The following are equivalent:

(i) The function $\psi$ belongs to $L^{2}\left((1,+\infty), \frac{d u}{u^{1+2 r}}\right)$.

(ii) The function $t \longmapsto L(r+i t) \hat{\varphi}(r+i t)$ belongs to $L^{2}(\mathbb{R})$.

We will see in Corollary 3.4 that conditions (i) and (ii) above are satisfied whenever $r>1$. Furthermore, Theorem 2.1 is a generalization of dR07a, Proposition 3.3 ] when $\varphi=\chi_{(0,1)}$ and $r=1 / 2$.

We see that the second condition of the theorem above depends on some growth estimates of $L$ in a vertical strip which is, in its turn, linked with questions related to the convexity bound and to the Lindelöf hypothesis. In particular, if $L$ is a function in the Selberg class, then one can prove that $L$ satisfies the generalized Lindelöf hypothesis if and only if for every $k \in \mathbb{N}$, we have

$$
t \longmapsto \frac{L^{k}\left(\frac{1}{2}+i t\right)}{\frac{1}{2}+i t} \in L^{2}(\mathbb{R}) .
$$

Note that with the special choice of $\varphi=\chi_{(0,1)}$, then $\hat{\varphi}(s)=1 / s$ and condition (ii) above for $r=1 / 2$ means exactly that (2.1) is satisfied for $k=1$. Moreover, in dR07a, using the functional equation, it is shown that the condition $\psi \in L^{2}\left((1,+\infty), \frac{d u}{u^{2}}\right)$ (in the case $\left.\varphi=\chi_{(0,1)}\right)$ is necessary for the generalized Riemann Hypothesis for $L$-functions in the Selberg class to hold. In dR07b, it is also shown that the condition on $\psi$ (still with $\left.\varphi=\chi_{(0,1)}\right)$ is satisfied for $L$-functions in the Selberg class of degree less than 4 .

Fix an integer $m \geq 0$ and let $W=\bigcup_{n \geq 1}(0,1]^{n}$. We say that $\alpha \in W$ is of length $n$ if $\alpha$ belongs to $(0,1]^{n}$. For each $\alpha$ in $W$, its length is denoted by $\ell(\alpha)$. Now let $\alpha \in W$ and $c \in \mathbb{C}^{\ell(\alpha)}$. We say that $A=(\alpha, c)$ is an $m$-admissible sequence if

$$
\sum_{j=1}^{\ell(\alpha)} c_{j} \alpha_{j}\left(\log \alpha_{j}\right)^{k}=0 \text { for all } 0 \leq k \leq m-1 .
$$

Furthermore, $A$ is said to be nontrivial if $c \in \mathbb{C}^{\ell(\alpha)} \backslash\{(0, \ldots, 0)\}$.

It is easy to prove (see Lemma 4.2 and Lemma 4.1) that for any fixed $\ell>m$, there are infinitely many nontrivial $m$-admissible sequences of length $\ell$. We also easily see that every $A=(\alpha, c)$ is 0 -admissible. Note that the notion of admissible sequences had been introduced in [R07a] and it is a generalization of condition (1.1) which appears in Nyman's theorem and which corresponds to $m=1$. 
From now on, we assume that $r$ is chosen such that

$$
\psi \in L^{2}\left((1,+\infty), \frac{d u}{u^{1+2 r}}\right) .
$$

Then we associate to each $m_{L}$-admissible sequence $A=(\alpha, c)$ the function $f_{A, r}$ defined by

$$
f_{A, r}(t)=t^{r-\sigma_{0}} \sum_{j=1}^{\ell(\alpha)} c_{j} \psi\left(\frac{\alpha_{j}}{t}\right), \quad t>0 .
$$

We will show that $f_{A, r} \in L_{*}^{2}\left((0,1), \frac{d t}{t^{1-2 \sigma_{0}}}\right)$ and we let

$$
K_{r}=\operatorname{span}\left\{f_{A, r}: A \text { a } m_{L} \text {-admissible sequence }\right\}
$$

and

$$
d_{r}(\lambda)=\operatorname{dist}\left(t^{\bar{\lambda}-2 \sigma_{0}} \chi_{(0,1)}, K_{r}\right) \quad\left(\lambda \in \Pi_{\sigma_{0}}\right),
$$

where the (closed) span and the distance are taken with respect to the space $L_{*}^{2}\left((0,1), \frac{d t}{t^{1-2 \sigma_{0}}}\right)$. Let us remark that we trivially have $d_{r}^{2}(\lambda) \leq 1 /\left(2 \Re(\lambda)-2 \sigma_{0}\right)$.

One of our main theorems is the following, which gives zero-free discs for the function $L$.

Theorem 2.2. Let $\lambda \in \Pi_{\sigma_{0}}$. Then $L$ does not vanish on $r-\sigma_{0}+D_{r}(\lambda)$, where

$$
D_{r}(\lambda):=\left\{\mu \in \mathbb{C}:\left|\frac{\mu-\lambda}{\mu+\bar{\lambda}-2 \sigma_{0}}\right|<\sqrt{1-2\left(\Re(\lambda)-\sigma_{0}\right) d_{r}^{2}(\lambda)}\right\} .
$$

Note that the zero-free regions which appear in Theorem 2.2 are discs (empty if $\left.d_{r}^{2}(\lambda)=1 /\left(2 \Re(\lambda)-2 \sigma_{0}\right)\right)$ or half-planes. More precisely, for $\lambda=a+i b \in \Pi_{\sigma_{0}}$ $\left(a>\sigma_{0}, b \in \mathbb{R}\right)$ and $R \in[0,1]$, then the set

$$
\left\{\mu \in \mathbb{C}:\left|\frac{\mu-\lambda}{\mu+\bar{\lambda}-2 \sigma_{0}}\right|<R\right\}
$$

is the open (Euclidean) disc whose center is $\Omega=\left(\frac{a+R^{2}\left(a-2 \sigma_{0}\right)}{1-R^{2}}, b\right)$ and radius is $\frac{2 R\left(a-\sigma_{0}\right)}{1-R^{2}}$ if $R \in\left[0,1\right.$ [; if $R=1$ this set is the half-plane $\Pi_{\sigma_{0}}$. In both cases, we easily see that this set is contained in the half-plane $\Pi_{\sigma_{0}}$ (recall that by definition of $\sigma_{0}$, the function $s \mapsto L(s)$ has an analytic continuation to $\left.\Pi_{\sigma_{0}}\right)$.

As a corollary of the proof of Theorem 2.2, we obtain another explicit version.

Corollary 2.3. Let $\lambda \in \Pi_{\sigma_{0}}$. Then $L$ does not vanish on the disc

$$
r-\sigma_{0}+\left\{\mu \in \mathbb{C}:\left|\frac{\mu-\lambda}{\mu+\bar{\lambda}-2 \sigma_{0}}\right|<\sqrt{2\left(\Re(\lambda)-\sigma_{0}\right)} \frac{\left|\widehat{f_{A, r}}(\lambda)\right|}{\left\|f_{A, r}\right\|_{2}}\right\},
$$

for any $m_{L}$-admissible sequence $A$.

Note that taking $L(s)=\zeta(s)$ and $\varphi=\chi_{(0,1)}$ (so that $\sigma_{0}=\sigma_{1}=0$ ) we recover exactly the results of Nikolski ([Nik95]). Now, taking $\varphi(t)=(1-t)^{-\sigma_{1}} \chi_{(0,1)}(t)$, we obtain larger zero-free discs whenever $\Im(\lambda)$ is large enough. We refer the reader to Section 7 for further discussions about the Rieman zeta function and other examples. 
We will see in Theorem 4.3 that

$$
\widehat{f_{A, r}}(\lambda)=L\left(\lambda+r-\sigma_{0}\right) \hat{\varphi}\left(\lambda+r-\sigma_{0}\right)\left(\sum_{j=1}^{n} c_{j} \alpha_{j}^{\lambda+r-\sigma_{0}}\right), \quad \lambda \in \Pi_{\sigma_{0}} .
$$

Therefore Corollary 2.3 can be understood as follows: let $\lambda \in \Pi_{\sigma_{0}}$ such that $L\left(\lambda+r-\sigma_{0}\right) \neq 0$; then there is a small neighborhood of $\lambda+r-\sigma_{0}$ free of zeros for $L$. Of course, this is an obvious consequence of the continuity of the function $L$ but the interest of Corollary 2.3 is that it gives an explicit neighborhood where the function $L$ does not vanish; moreover, this explicit neighborhood is expressed in terms of the values of $L$ and, in particular, it does not use any estimates of the derivatives.

Finally, we obtain a general Beurling-Nyman type theorem.

Theorem 2.4. Suppose that the function $\hat{\varphi}$ does not vanish on the half-plane $\Pi_{r}$, that $\lim \sup _{x \rightarrow+\infty} \frac{\log \left|\hat{\varphi}\left(x+r-\sigma_{0}\right)\right|}{x}=0$ and that $a_{1} \neq 0$. Then the following assertions are equivalent:

(1) The function $L$ does not vanish on the half-plane $\Pi_{r}$.

(2) There exists $\lambda \in \Pi_{\sigma_{0}}$ such that $d_{r}(\lambda)=0$.

(3) For all $\lambda \in \Pi_{\sigma_{0}}$, we have $d_{r}(\lambda)=0$.

(4) We have $K_{r}=L_{*}^{2}\left((0,1), d t / t^{1-2 \sigma_{0}}\right)$.

Taking $L(s)=\zeta(s), r=\lambda=1 / 2$ and $\varphi=\chi_{(0,1)}$ (so that we can take $\sigma_{0}=\sigma_{1}=$ $0)$, we obtain Nyman's theorem. Now, taking $\varphi(t)=(1-t)^{-\sigma_{1}} \chi_{(0,1)}(t)$ we extend the results of dR07a] (see also Section 7 for more details).

Another consequence of Theorem 2.2 is, in a way, a Beurling-Nyman criterion for Dirichlet $L$-functions. More precisely, let $\chi$ be a Dirichlet character with conductor $q$ and $L(\chi, s)$ its $L$-function. Then, for $1 / 2 \leq r<1$, we define $d_{r}$ by

$$
d_{r}=\min _{\ell, c, \alpha}\left(\int_{0}^{1}\left|t^{1-r}-t^{r} \sum_{j=1}^{\ell} c_{j} \sum_{n<\alpha_{j} / t} \chi(n)\right|^{2} \frac{d t}{t}\right)^{\frac{1}{2}}
$$

where the minimum is taken over all $\ell \geq 0, c=\left(c_{j}\right) \in \mathbb{C}^{\ell}$ and $\alpha=\left(\alpha_{j}\right) \in(0,1]^{\ell}$. One can show (see Proposition 7.5) that $d_{r}^{2}<1 /(2-2 r)$. We have

Theorem 2.5. If

$$
d_{r}^{2} \leq \frac{1}{2-2 r}-\frac{C^{2}}{2(\log q)^{2}(1-r)^{3}}
$$

for some constant $C>0$ and some $1 / 2 \leq r \leq 1$, then $L(\chi, \sigma)$ does not vanish in the real interval $\sigma>1-C / \log q$.

If we could take $C$ independent of $\chi$ in (2.4), then Theorem 2.5 would solve the Siegel zero problem.

In order to obtain the criterion for the Siegel zero problem, we consider all Dirichlet characters $\chi$ and an absolute constant $C$ independent of $\chi$.

The next section is devoted to the proof of Theorem 2.1 and to the study of the function $\psi$. Section 4 will focus on the admissible sequences and the functions $f_{A, r}$. Theorem 2.2 and Corollary 2.3 will be proven in Section 5, and Theorem 2.4 will be proven in Section 6. Some explicit examples will be studied in Section 7 in which we will prove Theorem 2.5 . 


\section{The Function $\boldsymbol{\psi}$ And Proof of Theorem 2.1}

We define the functions $\psi_{1}$ and $\psi_{2}$ by

$$
\begin{aligned}
& \psi_{1}(u)=\operatorname{res}\left(L(s) \hat{\varphi}(s) u^{s}, s=1\right) \quad\left(u \in \mathbb{R}_{+}\right) \\
& \psi_{2}(u)=\sum_{n<u} a_{n} \varphi\left(\frac{n}{u}\right) \quad\left(u \in \mathbb{R}_{+}\right)
\end{aligned}
$$

so that $\psi(u)=\psi_{1}(u)-\psi_{2}(u)$ by definition.

The function $s \mapsto \hat{\varphi}(s)$ is analytic in $\Pi_{\sigma_{0}}$, and the meromorphic continuation of $L(s)$ has a pole (of order $m_{L}$ ) only at $s=1$; hence we can write

$$
L(s) \hat{\varphi}(s)=\sum_{k=1}^{m_{L}} \frac{p_{-k}}{(s-1)^{k}}-H(s),
$$

with $H$ analytic in $\Pi_{\sigma_{0}}$.

Lemma 3.1. We have

$$
H(s)=\int_{0}^{1} \psi\left(\frac{1}{t}\right) t^{s-1} d t=\hat{\phi}(s), \quad \Re(s)>1,
$$

where $\phi(t)=\psi(1 / t) \chi_{(0,1)}(t)$.

Proof of Lemma 3.1. On the one hand, we have

$$
L(s) \hat{\varphi}(s)=\int_{1}^{+\infty} \psi_{2}(u) u^{-s-1} d u, \quad \Re(s)>1 .
$$

On the other hand, the equality

$$
\int_{1}^{+\infty} \psi_{1}(u) u^{-s-1} d u=\sum_{k=1}^{m_{L}} \frac{p_{-k}}{(s-1)^{k}}
$$

follows by linearity using the classical identities

$$
\operatorname{res}\left(\frac{u^{s}}{(s-1)^{k}}, s=1\right)=\frac{u(\log u)^{k-1}}{(k-1) !}
$$

and

$$
\frac{1}{(k-1) !} \int_{1}^{+\infty}(\log u)^{k-1} u^{-s} d u=\frac{1}{(s-1)^{k}} .
$$

Equations (3.3) and (3.4) now imply the expected result.

Remark 3.2. From (3.1) and (3.5), we easily see that we can write $\psi_{1}(t)=t P(\log t)$, where $P$ is a polynomial of degree $<m_{L}\left(P \equiv 0\right.$ if $\left.m_{L}=0\right)$. More precisely we have

$$
\psi_{1}(t)=t \sum_{k=1}^{m_{L}} \frac{p_{-k}}{(k-1) !}(\log t)^{k-1} .
$$

Lemma 3.3. The function $s \mapsto H(s)$ is of finite order on $\Pi_{r}$. Moreover, for all $\sigma>1$, the function $t \mapsto H(\sigma+i t)$ belongs to $L^{2}(\mathbb{R})$ and

$$
\int_{\mathbb{R}}|H(\sigma+i t)|^{2} d t=O\left(\frac{1}{\sigma^{1-2 \sigma_{1}}}\right) \quad \text { for } \sigma \rightarrow+\infty .
$$


Proof of Lemma 3.3. The function $s \mapsto H(s)$ is bounded on a neighborhood $V_{1}$ of $s=1$. The functions $s \mapsto \sum_{j} \frac{p_{-j}}{(s-1)^{j}}$ and (by assumption) $s \mapsto L(s)$ are of finite order on $\Pi_{\sigma_{0}} \backslash V_{1}$. Moreover, since $\varphi(x)=O\left(x^{-\sigma_{0}}(1-x)^{-\sigma_{1}}\right)$ and $r>\sigma_{0}$, it is clear that $s \mapsto \hat{\varphi}(s)$ is bounded on the closure of $\Pi_{r}$. Hence we can conclude that $H$ is of finite order on $\Pi_{r}$, which proves the first part of the lemma.

Let $\sigma>1$ be a fixed real number. We easily check that, for every $j \geq 1$, the function $t \longmapsto(\sigma-1+i t)^{-j}$ belongs to $L^{2}(\mathbb{R})$ and we have

$$
\int_{\mathbb{R}}\left|\frac{1}{(\sigma+i t-1)^{j}}\right|^{2} d t=O\left(\frac{1}{\sigma^{2 j-1}}\right), \quad \sigma \rightarrow+\infty .
$$

Now, Plancherel's formula and the estimate $\varphi(x)=O\left(x^{-\sigma_{0}}(1-x)^{-\sigma_{1}}\right)$ yield

$$
\begin{aligned}
\int_{\mathbb{R}}|\hat{\varphi}(\sigma+i t)|^{2} d t & =2 \pi \int_{0}^{1}|\varphi(x)|^{2} x^{2 \sigma} \frac{d x}{x} \\
& =O\left(\int_{0}^{1} x^{2 \sigma-2 \sigma_{0}-1}(1-x)^{-2 \sigma_{1}} d x\right) .
\end{aligned}
$$

Then, the classical identity

$$
\beta\left(A, 1-2 \sigma_{1}\right):=\int_{0}^{1} x^{A-1}(1-x)^{1-2 \sigma_{1}} d x=\frac{\Gamma(A) \Gamma\left(1-2 \sigma_{1}\right)}{\Gamma\left(A+1-2 \sigma_{1}\right)}
$$

and Stirling's formula give

$$
\int_{0}^{1} x^{A-1}(1-x)^{1-2 \sigma_{1}} d x=O\left(\frac{1}{A^{1-2 \sigma_{1}}}\right), \quad A \rightarrow+\infty,
$$

and we can conclude the proof (recall that $s \mapsto L(s)$ is bounded in $\Pi_{\sigma}$ ).

Now we can prove Theorem 2.1.

Assume that (i) is satisfied. Then the function $\phi(t)=\psi\left(\frac{1}{t}\right) \chi_{(0,1)}(t)$ belongs to $L_{*}^{2}\left((0,1), \frac{d t}{t^{1-2 r}}\right)$, and thus the function $G:=\hat{\phi}$ belongs to $H^{2}\left(\Pi_{r}\right)$. The analytic continuation principle implies that the equality (3.2) is satisfied for every $s \in \Pi_{r}$, that is, $H(s)=\hat{\phi}(s)=G(s), s \in \Pi_{r}$. Since $G \in H^{2}\left(\Pi_{r}\right)$, we know that the function $G^{*}$, defined by

$$
G^{*}(t):=\lim _{\sigma \rightarrow r} G(\sigma+i t),
$$

exists almost everywhere on $\mathbb{R}$ and belongs to $L^{2}(\mathbb{R})$. But

$$
G^{*}(t)=\lim _{\sigma \rightarrow r} H(\sigma+i t)=H(r+i t)
$$

because $H$ is continuous on the closed half-plane $\Re(s) \geq r$. Therefore $t \longmapsto H(r+i t)$ belongs to $L^{2}(\mathbb{R})$. It remains to notice that for every $1 \leq k \leq m_{L}$, the function $t \longmapsto(r-1+i t)^{-k}$ belongs to $L^{2}(\mathbb{R})$ (this is where we have to assume that $r \neq 1$ whenever $\left.m_{L} \geq 1\right)$. Thus according to (3.1), we get that $t \mapsto L(r+i t) \hat{\varphi}(r+i t)$ belongs to $L^{2}(\mathbb{R})$.

Conversely assume that (ii) is satisfied. Then the function $t \longmapsto H(r+i t)$ belongs to $L^{2}(\mathbb{R})$.

Let $\sigma_{2}>\max (1, r)$. The function $H$ is analytic on $\Omega:=\left\{s: r<\Re(s)<\sigma_{2}\right\}$ and continuous on the closure of $\Omega$. Using the Hardy, Littlewood and Ingham theorem 
[HIP27, Theorem 7], we deduce from Lemma 3.3 that

$$
\sup _{r \leq \sigma \leq \sigma_{2}} \int_{\mathbb{R}}|H(\sigma+i t)|^{2} d t<+\infty .
$$

Hence, the second part of Lemma 3.3 gives that $H$ belongs to $H^{2}\left(\Pi_{r}\right)$. Since we have $H^{2}\left(\Pi_{r}\right)=\mathcal{M}\left(L_{*}^{2}\left((0,1), \frac{d t}{t^{1-2 r}}\right)\right)$, there exists a function $\phi_{1} \in L_{*}^{2}\left((0,1), \frac{d t}{t^{1-2 r}}\right)$ such that $H(s)=\hat{\phi}_{1}(s)$, for every $s \in \Pi_{r}$. Thus for $\Re(s)>\max (1, r)$, we have $\hat{\phi}_{1}(s)=H(s)=\hat{\phi}(s)$.

By injectivity of the Mellin transform, we get that

$$
\psi\left(\frac{1}{t}\right) \chi_{(0,1)}(t)=\phi(t)=\phi_{1}(t)
$$

Thus $t \longmapsto \psi\left(\frac{1}{t}\right) \chi_{(0,1)}(t)$ belongs to $L_{*}^{2}\left((0,1), \frac{d t}{t^{1-2 r}}\right)$, which implies that $\psi$ belongs to $L^{2}\left((1,+\infty), \frac{d u}{u^{1+2 r}}\right)$ and that concludes the proof of Theorem 2.1.

Corollary 3.4. Let $r>1$. Then $\psi \in L^{2}\left((1,+\infty), \frac{d u}{u^{1+2 r}}\right)$ and we have

$$
\|\psi\|_{2}=O\left(r^{\sigma_{1}-1 / 2}\right) \text {, as } r \rightarrow+\infty \text {. }
$$

Proof. Let $r>1$ be a fixed real number. By Lemma 3.3, the function $t \longmapsto H(r+i t)$ belongs to $L^{2}(\mathbb{R})$ and for every $1 \leq j \leq m_{L}$, the function $t \longmapsto(r-1+i t)^{-j}$ also belongs to $L^{2}(\mathbb{R})$. Therefore it follows from (3.1) that $t \longmapsto L(r+i t) \hat{\varphi}(r+i t)$ belongs to $L^{2}(\mathbb{R})$, and Theorem 2.1 implies that $\psi \in L^{2}\left((1,+\infty), \frac{d u}{u^{1+2 r}}\right)$. Moreover, if we let $\phi(t)=\psi(1 / t) \chi_{(0,1)}(t)$ as before, we have by Plancherel's formula

$$
\begin{aligned}
\|\psi\|_{2}^{2} & =\int_{1}^{+\infty}|\psi(u)|^{2} \frac{d u}{u^{1+2 r}}=\int_{0}^{1}\left|\psi\left(\frac{1}{t}\right)\right|^{2} \frac{d t}{t^{1-2 r}} \\
& =\|\phi\|_{L_{*}^{2}\left((0,1), \frac{d t}{t^{1-2 r}}\right)}^{2} \\
& =\frac{1}{2 \pi} \int_{\mathbb{R}}|\hat{\phi}(r+i t)|^{2} d t \\
& =\frac{1}{2 \pi} \int_{\mathbb{R}}|H(r+i t)|^{2} d t
\end{aligned}
$$

and Lemma 3.3 gives the result.

Remark 3.5. In fact, if the coefficients of $L(s)$ have some nice arithmetical properties, for example so that a Wiener-Ikehara type theorem can be applied to $L(s)$, one can expect that the main contributions of $\psi_{1}$ will be compensated by the main contributions of $\psi_{2}$ and so that the function $\psi$ will belong to the space $L^{2}\left((1,+\infty), \frac{d u}{u^{1+2 r}}\right)$ for smaller values of $r$. For example, if $L(s)=\zeta(s)$ and if $\varphi(t)=\chi_{0,1}(t)$ for all $t \in(0,1)$, then $\psi(u)=u-\lceil u\rceil+1$ (so $\psi(u)=\{u\}$ for almost all $u \in(1, \infty))$ and $\psi \in L^{2}\left((1,+\infty), \frac{d u}{u^{1+2 r}}\right)$ for all $r>0$. In this case $\hat{\varphi}(s)=1 / s$ and $t \mapsto|\zeta(r+i t)| /|r+i t| \in L^{2}(\mathbb{R})$ for all $r>0$ as expected by Theorem 2.1. We will discuss other examples in Section 7

Remark 3.6. Assume that $\varphi$ is bounded at $t=1$ (which corresponds to $\sigma_{1}=0$ ). Thus, for every $\alpha>1$, we have $\psi(t)=\psi_{1}(t)-\psi_{2}(t)=O\left(t^{\alpha}\right), t \rightarrow+\infty$. Indeed, on the one hand, $\psi_{1}(t)=t P(\log t)$, where $P$ is a polynomial of degree $<m_{L}$. On the other hand, let $\alpha>1$ be a fixed real number. Then there exists a constant $C>0$ such that

$$
|\varphi(t)| \leq C t^{-\alpha}, \quad t \in(0,1)
$$


Hence we get

$$
\begin{aligned}
\left|\psi_{2}(t)\right| & \leq \sum_{n<t}\left|a_{n}\right|\left|\varphi\left(\frac{n}{t}\right)\right| \leq C \sum_{n<t}\left|a_{n}\right|\left(\frac{t}{n}\right)^{\alpha} \\
& \leq C^{\prime} t^{\alpha} \sum_{n<t} \frac{1}{n^{\alpha-\varepsilon}},
\end{aligned}
$$

which gives that $\psi_{2}(t)=O\left(t^{\alpha}\right)$ as $t \rightarrow+\infty$. It is now easy to check that $\psi$ belongs to $L^{2}\left((1,+\infty), \frac{d u}{u^{1+2 r}}\right)$, for every $r>1$ and $\|\psi\|_{2}=O\left(r^{-1 / 2}\right), r \rightarrow+\infty$. We recover Corollary 3.4 .

\section{Admissible sequences and the functions $\boldsymbol{f}_{\boldsymbol{A}, \boldsymbol{r}}$}

Given $\ell \geq 1, m \geq 1, \alpha \in(0,1]^{\ell}$ and $c \in \mathbb{C}^{\ell}$, recall that $A=(\alpha, c)$ is said to be an $m$-admissible sequence of length $\ell$ if

$$
\sum_{j=1}^{\ell} c_{j} \alpha_{j}\left(\log \alpha_{j}\right)^{k}=0, \quad \text { for all } 0 \leq k \leq m-1 .
$$

The following results will be useful in the sequel.

Lemma 4.1. Let $(\alpha, c)$ be an m-admissible sequence.

(1) For any polynomial $P$ of degree $d<m$, for any real $t>0$, we have

$$
\sum_{j=1}^{\ell(\alpha)} c_{j} \alpha_{j} P\left(\log \left(\alpha_{j} / t\right)\right)=0 .
$$

(2) For any positive real number $\lambda_{1}$ and for any real number $\lambda_{2} \geq \max _{j}\left(\alpha_{j}\right)$, the sequences $\left(\left(\alpha_{j}^{\lambda_{1}}\right)_{j},\left(c_{j} \alpha_{j}^{1-\lambda_{1}}\right)_{j}\right)$ and $\left(\left(\alpha_{j} / \lambda_{2}\right)_{j}, c\right)$ are both $m$-admissible sequences.

Proof. (1) If $t=1$, the equality comes from the definition. If $t>0$, we apply the result for $t=1$ to the polynomial $P_{t}(X)=P(X-\log t)$. The rest of the proof follows immediately from the definition.

For an $m$-admissible sequence $A=(\alpha, c)$ we define the entire function

$$
g_{A}(s)=\sum_{j=1}^{\ell(\alpha)} c_{j} \alpha_{j}^{s} \quad(s \in \mathbb{C}) .
$$

Using (2.2), we notice that these functions satisfy

$$
g_{A}^{(k)}(1)=0 \quad(0 \leq k \leq m-1) .
$$

Lemma 4.2. Let $m$ be a nonnegative integer. Then we have the following:

(1) For every integer $\ell \geq m+1$ and every $0<\alpha_{1}<\alpha_{2}<\cdots<\alpha_{\ell} \leq 1$, there exists $c \in \mathbb{C}^{\ell}$ such that $A=(\alpha, c)$ is an $m$-admissible sequence and $g_{A}^{(m)}(1) \neq 0$. Furthermore we can choose $c_{\ell} \neq 0$.

(2) For every $s_{1} \in \mathbb{C} \backslash\{1\}$, there exists an $m$-admissible sequence $A$ such that $g_{A}\left(s_{1}\right) \neq 0$. 
Proof. (1) Let $\ell \geq m+1$. Then there is a unique vector $\left(b_{1}, b_{2}, \ldots, b_{m}, b_{\ell}\right) \in \mathbb{C}^{m+1}$ such that

$$
\left(\begin{array}{ccccc}
1 & \ldots & \ldots & 1 & 1 \\
\left(\log \alpha_{1}\right) & \ldots & \ldots & \left(\log \alpha_{m}\right) & \left(\log \alpha_{\ell}\right) \\
\vdots & & & \vdots & \vdots \\
\left(\log \alpha_{1}\right)^{m} & \ldots & \ldots & \left(\log \alpha_{m}\right)^{m} & \left(\log \alpha_{\ell}\right)^{m}
\end{array}\right)\left(\begin{array}{c}
b_{1} \\
\vdots \\
b_{m} \\
b_{\ell}
\end{array}\right)=\left(\begin{array}{c}
0 \\
\vdots \\
0 \\
1
\end{array}\right)
$$

since the corresponding van der Monde matrix is invertible. Setting

$$
c_{j}= \begin{cases}\frac{b_{j}}{\alpha_{j}}, & 1 \leq j \leq m, \\ 0, & m+1 \leq j \leq \ell-1, \\ \frac{b_{\ell}}{\alpha_{\ell}}, & j=\ell,\end{cases}
$$

then $A=(\alpha, c)$ is an $m$-admissible sequence and $g_{A}^{(m)}(1)=1$. Furthermore we see that $b_{\ell} \neq 0$ (otherwise, we would easily have $b_{1}=b_{2}=\cdots=b_{m}=b_{\ell}=0$, which contradicts (4.2)); therefore $c_{\ell} \neq 0$.

(2) Let $A=(\alpha, c)$ be a nontrivial $m$-admissible sequence.

For $0<\lambda<1$, consider $g_{A_{\lambda}}(s)=g_{A}(1-\lambda+\lambda s)$. Note that

$$
g_{A_{\lambda}}(s)=\sum_{j=1}^{\ell} c_{j} \alpha_{j}^{1-\lambda}\left(\alpha_{j}^{\lambda}\right)^{s},
$$

and, by Lemma 4.1 $A_{\lambda}=\left(\left(\alpha_{j}^{\lambda}\right)_{j},\left(c_{j} \alpha_{j}^{1-\lambda}\right)_{j}\right)$ is an $m$-admissible sequence. Now let $s_{1} \neq 1$; then there necessarily exists $0<\lambda<1$ such that $g_{A_{\lambda}}\left(s_{1}\right) \neq 0$, since $g_{A}$ is analytic and not identically zero.

Theorem 4.3. Let $r>\sigma_{0}$ such that $\psi \in L^{2}\left((1,+\infty), \frac{d u}{u^{1+2 r}}\right)$ and let $A=(\alpha, c)$ be an $m_{L}$-admissible sequence. We define

$$
f_{A, r}(t)=t^{r-\sigma_{0}} \sum_{j=1}^{\ell(\alpha)} c_{j} \psi\left(\frac{\alpha_{j}}{t}\right) \quad(t>0) .
$$

Then we have:

(1) $f_{A, r}(t)=0$ if $t>\max _{j} \alpha_{j}$.

(2) $f_{A, r}(t) \in L_{*}^{2}\left((0,1), \frac{d t}{t^{1-2 \sigma_{0}}}\right)$.

(3) For $\Re(s)>\sigma_{0}$, we have

$$
\widehat{f_{A, r}}(s)=-L\left(s+r-\sigma_{0}\right) \hat{\varphi}\left(s+r-\sigma_{0}\right) g_{A}\left(s+r-\sigma_{0}\right) .
$$

Proof. (1) We write $\psi=\psi_{1}-\psi_{2}$ with $\psi_{1}(u)=\operatorname{res}\left(L(s) \hat{\varphi}(s) u^{s}, s=1\right)$ and $\psi_{2}(u)=$ $\sum_{n<u} a_{n} \varphi\left(\frac{n}{u}\right)$. From Remark 3.2. we have $\psi_{1}(u)=u P(\log u)$, where $P$ is a polynomial of degree $<m_{L}$. Then

$$
\begin{aligned}
\sum_{j=1}^{\ell(\alpha)} c_{j} \psi\left(\frac{\alpha_{j}}{t}\right) & =\sum_{j=1}^{\ell(\alpha)} c_{j} \psi_{1}\left(\frac{\alpha_{j}}{t}\right)-\sum_{j=1}^{\ell(\alpha)} c_{j} \psi_{2}\left(\frac{\alpha_{j}}{t}\right) \\
& =\sum_{j=1}^{\ell(\alpha)} c_{j} \frac{\alpha_{j}}{t} P\left(\log \left(\frac{\alpha_{j}}{t}\right)\right)-\sum_{j=1}^{\ell(\alpha)} c_{j} \sum_{n<\alpha_{j} / t} a_{n} \varphi\left(\frac{n t}{\alpha_{j}}\right) .
\end{aligned}
$$

If $t>\max _{j}\left(\alpha_{j}\right)$ the second sum is 0 because $\alpha_{j} / t<1$. Furthermore, by Lemma 4.1 (1), the first sum is also zero. 
(2) We first prove that each term $t^{r-\sigma_{0}} \psi\left(\frac{\alpha_{j}}{t}\right)$ belongs to the space $L^{2}((0,\|\alpha\|)$, $\left.\frac{d t}{t^{1-2 \sigma_{0}}}\right)$, where $\|\alpha\|:=\max _{j} \alpha_{j}$. Indeed, we have

$$
\int_{0}^{\|\alpha\|} t^{2 r-2 \sigma_{0}}\left|\psi\left(\frac{\alpha_{j}}{t}\right)\right|^{2} \frac{d t}{t^{1-2 \sigma_{0}}}=\alpha_{j}^{2 r} \int_{\frac{\alpha_{j}}{\|\alpha\|}}^{+\infty}|\psi(u)|^{2} \frac{d u}{u^{1+2 r}}
$$

and

$$
\int_{\frac{\alpha_{j}}{\|\alpha\|}}^{+\infty}|\psi(u)|^{2} \frac{d u}{u^{1+2 r}}=\int_{\frac{\alpha_{j}}{\|\alpha\|}}^{1}\left|\psi_{1}(u)\right|^{2} \frac{d u}{u^{1+2 r}}+\int_{1}^{+\infty}|\psi(u)|^{2} \frac{d u}{u^{1+2 r}}
$$

since $\psi(u)=\psi_{1}(u)$ for $u<1$. Now, by hypothesis, the second integral is finite and the first integral is also finite because $\psi_{1}(u)=u P(\log u)$ and $\frac{\alpha_{j}}{\|\alpha\|}>0$. Since $f_{A, r}(t)=0$ for $t>\|\alpha\|$, the expected result follows by linearity. Furthermore, we see that

$$
\begin{aligned}
& \left\|f_{A, r}\right\|_{L_{*}^{2}\left((0,1), \frac{d t}{t^{1-2 \sigma_{0}}}\right)} \\
& \quad \leq \sum_{j=1}^{\ell(\alpha)}\left|c_{j} \alpha_{j}^{r}\right|\left(\left(\int_{\frac{\min _{j} \alpha_{j}}{\max _{j} \alpha_{j}}}^{1}\left|\psi_{1}(u)\right|^{2} \frac{d u}{u^{1+2 r}}\right)^{1 / 2}+\|\psi\|_{L^{2}\left((1,+\infty), \frac{d u}{u^{1+2 r}}\right)}\right) .
\end{aligned}
$$

(3) For $\Re(s)>1$, we have by (3.3),

$$
L(s) \hat{\varphi}(s)=\int_{1}^{+\infty} \psi_{2}(u) u^{-s-1} d u
$$

Hence

$$
\begin{aligned}
L(s) \hat{\varphi}(s) \sum_{j=1}^{\ell(\alpha)} c_{j} \alpha_{j}^{s} & =\sum_{j=1}^{\ell(\alpha)} c_{j} \int_{1}^{+\infty}\left(\frac{u}{\alpha_{j}}\right)^{-s} \psi_{2}(u) \frac{d u}{u} \\
& =\sum_{j=1}^{\ell(\alpha)} c_{j} \int_{0}^{\alpha_{j}} \psi_{2}\left(\frac{\alpha_{j}}{t}\right) \frac{d t}{t^{1-s}} \\
& =\sum_{j=1}^{\ell(\alpha)} c_{j} \int_{0}^{1} \psi_{2}\left(\frac{\alpha_{j}}{t}\right) \frac{d t}{t^{1-s}}
\end{aligned}
$$

where the last equality follows from $\psi_{2}\left(\alpha_{j} / t\right)=0$ if $\alpha_{j}<t \leq 1$.

Using Lemma 4.1 (1) once more, we have

$$
\sum_{j=1}^{\ell(\alpha)} c_{j} \psi\left(\frac{\alpha_{j}}{t}\right)=-\sum_{j=1}^{\ell(\alpha)} c_{j} \psi_{2}\left(\frac{\alpha_{j}}{t}\right) \quad(t>0),
$$


whence

$$
\begin{aligned}
L(s) \hat{\varphi}(s) \sum_{j=1}^{\ell(\alpha)} c_{j} \alpha_{j}^{s} & =-\int_{0}^{1}\left(\sum_{j=1}^{\ell(\alpha)} c_{j} \psi\left(\frac{\alpha_{j}}{t}\right)\right) \frac{d t}{t^{1-s}} \\
& =-\int_{0}^{1} t^{\sigma_{0}-r} f_{A, r}(t) \frac{d t}{t^{1-s}} \\
& =-\int_{0}^{1} f_{A, r}(t) t^{s+\sigma_{0}-r-1} d t \\
& =-\widehat{f}_{A, r}\left(s+\sigma_{0}-r\right), \quad \Re(s)>1 .
\end{aligned}
$$

So, for $\Re(s)>1+\sigma_{0}-r$, we have $\widehat{f_{A, r}}(s)=-L\left(s+r-\sigma_{0}\right) \hat{\varphi}\left(s+r-\sigma_{0}\right) g_{A}\left(s+r-\sigma_{0}\right)$. By the analytic continuation principle, the equality holds for $\Re(s)>\sigma_{0}$. (Note that from (4.1) the pole of $L\left(s+r-\sigma_{0}\right)$ is killed by the zero of $g_{A}\left(s+r-\sigma_{0}\right)$ at $s=1-r+\sigma_{0}$.)

Remark 4.4. Let $m \geq 1$, and let $A=(\alpha, c)$ be an $m$-admissible sequence. Then, we have

$$
\sum_{j=1}^{\ell} c_{j} \psi\left(\frac{\alpha_{j}}{t}\right)=\sum_{j=1}^{\ell} c_{j}\left(\psi\left(\frac{\alpha_{j}}{t}\right)-\alpha_{j} \psi\left(\frac{1}{t}\right)\right) .
$$

Thus, if $m=1$ and

$$
K_{r}=\operatorname{span}\left(t^{r-\sigma_{0}} \sum_{j=1}^{\ell} c_{j} \psi\left(\frac{\alpha_{j}}{t}\right): A=(\alpha, c) \text { a 1-admissible sequence }\right),
$$

we easily check that

$$
K_{r}=\operatorname{span}\left(t^{r-\sigma_{0}}\left(\psi\left(\frac{\alpha}{t}\right)-\alpha \psi\left(\frac{1}{t}\right)\right): 0<\alpha \leq 1\right) .
$$

In particular, if $L(s)=\zeta(s), \varphi(t)=\chi_{(0,1)}(t)$ (that is, $\sigma_{1}=0$ ), we obtain that $\psi(u)=\{u\}$ for almost all $u \in(1,+\infty)$ and then $K_{r}=\tilde{K}_{r}$, the subspace introduced in Nik95].

\section{Zero-Free Regions For DiRICHlet SERIES}

Before proving Theorem 2.2 we need some well-known tools concerning the Hardy space $H^{2}\left(\Pi_{\sigma_{0}}\right)$. For these we refer to [Hof62, Chapter 8]. Actually, in Hof62, the following facts are stated for the space $H^{2}\left(\Pi_{0}\right)$, but it is easy to obtain the corresponding results for $H^{2}\left(\Pi_{\sigma_{0}}\right)$, for instance using the unitary map $h(z) \longmapsto$ $h\left(z-\sigma_{0}\right)$ from $H^{2}\left(\Pi_{\sigma_{0}}\right)$ onto $H^{2}\left(\Pi_{0}\right)$.

Recall that if $h \in H^{2}\left(\Pi_{\sigma_{0}}\right)$, then

$$
h^{*}\left(\sigma_{0}+i t\right):=\lim _{\substack{\sigma \rightarrow \sigma_{0} \\>}} h(\sigma+i t)
$$

exists for almost every $t \in \mathbb{R}$ (with respect to the Lebesgue measure). Moreover we have $h^{*} \in L^{2}\left(\sigma_{0}+i \mathbb{R}\right)$ and $\|h\|_{2}=\left\|h^{*}\right\|_{2}$. We can therefore identify (unitarily) $H^{2}\left(\Pi_{\sigma_{0}}\right)$ with a (closed) subspace of $L^{2}\left(\sigma_{0}+i \mathbb{R}\right)$. In the following we use the symbol $h$ not only for the function in $H^{2}\left(\Pi_{\sigma_{0}}\right)$ but also for its "radial" limit (in 
other words we forget the star). This identification enables us to consider $H^{2}\left(\Pi_{\sigma_{0}}\right)$ as a Hilbert space, with scalar product given by

$$
\langle h, g\rangle_{2}=\int_{-\infty}^{+\infty} h\left(\sigma_{0}+i t\right) \overline{g\left(\sigma_{0}+i t\right)} d t, \quad h, g \in H^{2}\left(\Pi_{\sigma_{0}}\right) .
$$

Now for $\lambda \in \Pi_{\sigma_{0}}$, we have the following integral representation:

$$
h(\lambda)=\frac{1}{2 \pi} \int_{-\infty}^{+\infty} \frac{h\left(\sigma_{0}+i t\right)}{\lambda-\sigma_{0}-i t} d t
$$

so that we can write, using (5.1),

$$
h(\lambda)=\left\langle h, k_{\lambda}\right\rangle_{2},
$$

where $k_{\lambda}$ is the function in $H^{2}\left(\Pi_{\sigma_{0}}\right)$ defined by

$$
k_{\lambda}(z):=\frac{1}{2 \pi} \frac{1}{z-2 \sigma_{0}+\bar{\lambda}}, \quad z \in \Pi_{\sigma_{0}} .
$$

The function $k_{\lambda}$ is called the reproducing kernel of $H^{2}\left(\Pi_{\sigma_{0}}\right)$, and we have

$$
\left\|k_{\lambda}\right\|_{2}^{2}=\left\langle k_{\lambda}, k_{\lambda}\right\rangle_{2}=k_{\lambda}(\lambda)=\frac{1}{4 \pi\left(\Re(\lambda)-\sigma_{0}\right)} .
$$

In particular, the linear functional $h \longmapsto h(\lambda)$ is continuous on $H^{2}\left(\Pi_{\sigma_{0}}\right)$. Now recall a useful property of factorization for functions in the Hardy space.

Lemma 5.1. Let $h \in H^{2}\left(\Pi_{\sigma_{0}}\right)$ and $\mu \in \Pi_{\sigma_{0}}$ such that $h(\mu)=0$. Then there exists $g \in H^{2}\left(\Pi_{\sigma_{0}}\right)$ such that $\|h\|_{2}=\|g\|_{2}$ and

$$
h(z)=\frac{z-\mu}{z+\bar{\mu}-2 \sigma_{0}} g(z), \quad z \in \Pi_{\sigma_{0}} .
$$

Proof. See the results in Hof62, Chapter 8, p. 132] and apply the transform $h(z) \longmapsto h\left(z-\sigma_{0}\right)$.

Proof of Theorem 2.2. Denote by $E_{r}=\mathcal{M} K_{r}$, where $K_{r}$ is defined by (2.3). Since the Mellin transform is a unitary map from $L_{*}^{2}\left((0,1), \frac{d t}{t^{1-2 \sigma_{0}}}\right)$ onto $H^{2}\left(\Pi_{\sigma_{0}}\right)$, we have

$$
E_{r}=\operatorname{span}_{H^{2}\left(\Pi_{\sigma_{0}}\right)}\left(h_{A, r}: A \text { an } m_{L} \text {-admissible sequence) },\right.
$$

where $h_{A, r}(s)=\mathcal{M} f_{A, r}(s)$, for $\Re(s)>\sigma_{0}$. It follows from Theorem 4.3 that

$$
h_{A, r}(s)=-\frac{1}{\sqrt{2 \pi}} L\left(s+r-\sigma_{0}\right) \hat{\varphi}\left(s+r-\sigma_{0}\right) g_{A}\left(s+r-\sigma_{0}\right), \Re(s)>\sigma_{0} .
$$

Now assume that there is $\mu \in \Pi_{\sigma_{0}}$ such that $L\left(\mu+r-\sigma_{0}\right)=0$. Since $\mu \in \Pi_{\sigma_{0}}$, we get that $h_{A, r}(\mu)=0$, for every $m_{L}$-admissible sequence $A$. Thus, by continuity of $h \longmapsto h(\mu)$ in $H^{2}\left(\Pi_{\sigma_{0}}\right)$, we also have $h(\mu)=0$, for all $h \in E_{r}$. Since $h \in H^{2}\left(\Pi_{\sigma_{0}}\right)$, we know from Lemma 5.1 that there is $g \in H^{2}\left(\Pi_{\sigma_{0}}\right)$ such that $\|g\|_{2}=\|h\|_{2}$ and

$$
h(z)=\frac{z-\mu}{z+\bar{\mu}-2 \sigma_{0}} g(z) \quad\left(z \in \Pi_{\sigma_{0}}\right) .
$$

Hence with the Cauchy-Schwarz inequality and (5.2), we deduce that

$$
|h(\lambda)|=\left|\frac{\lambda-\mu}{\lambda+\bar{\mu}-2 \sigma_{0}}\right||g(\lambda)| \leq\left|\frac{\lambda-\mu}{\lambda+\bar{\mu}-2 \sigma_{0}}\right|\|g\|_{2}\left\|k_{\lambda}\right\|_{2},
$$


SO

$$
\sup _{\substack{h \in E_{r} \\\|h\|_{2}=1}}|h(\lambda)| \leq\left|\frac{\mu-\lambda}{\mu+\bar{\lambda}-2 \sigma_{0}}\right|\left\|k_{\lambda}\right\|_{2} .
$$

By contraposition, we have proved that $L$ does not vanish on

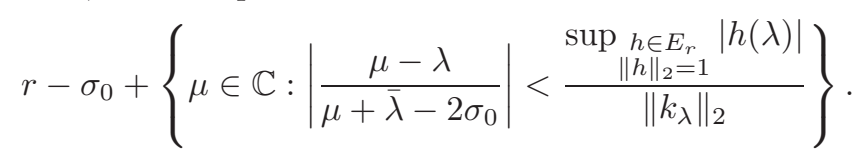

It remains to prove that

$$
\frac{\sup _{\|h\|_{2}=1}|h(\lambda)|^{2}}{\left\|k_{\lambda}\right\|_{2}^{2}}=1-2\left(\Re(\lambda)-\sigma_{0}\right) d_{r}^{2}(\lambda) .
$$

To show this equality, first observe that

$$
\sup _{\substack{h \in E_{r} \\\|h\|_{2}=1}}|h(\lambda)|=\sup _{\substack{h \in E_{r} \\\|h\|_{2}=1}}\left|\left\langle h, k_{\lambda}\right\rangle_{2}\right|=\left\|P_{E_{r}} k_{\lambda}\right\|_{2},
$$

where $P_{E}$ denotes the orthogonal projection onto the closed subspace $E$ of $H^{2}\left(\Pi_{\sigma_{0}}\right)$. Then Pythagoras' Theorem implies that

Hence

$$
\sup _{\substack{h \in E_{r} \\\|h\|_{2}=1}}|h(\lambda)|^{2}=\left\|P_{E_{r}} k_{\lambda}\right\|_{2}^{2}=\left\|k_{\lambda}\right\|_{2}^{2}-\left\|P_{E_{r}^{\perp}} k_{\lambda}\right\|_{2}^{2} .
$$

But for $\Re(s)>\sigma_{0}$, we have

$$
\frac{\sup _{\substack{h \in E_{r} \\\|h\|_{2}=1}}|h(\lambda)|^{2}}{\left\|k_{\lambda}\right\|_{2}^{2}}=1-\frac{\operatorname{dist}^{2}\left(k_{\lambda}, E_{r}\right)}{\left\|k_{\lambda}\right\|_{2}^{2}} .
$$

$$
\begin{aligned}
\mathcal{M}\left(t^{\bar{\lambda}-2 \sigma_{0}} \chi_{(0,1)}(t)\right)(s) & =\frac{1}{\sqrt{2 \pi}} \int_{0}^{1} t^{\bar{\lambda}-2 \sigma_{0}} t^{s-1} d t \\
& =\frac{1}{\sqrt{2 \pi}} \frac{1}{\bar{\lambda}-2 \sigma_{0}+s}=\sqrt{2 \pi} k_{\lambda}(s),
\end{aligned}
$$

according to (5.3) . Since the Mellin transform is an isometry from $L_{*}^{2}\left((0,1), \frac{d t}{t^{1-2 \sigma_{0}}}\right)$ onto $H^{2}\left(\Pi_{\sigma_{0}}\right)$, we obtain

$$
\operatorname{dist}\left(k_{\lambda}, E_{r}\right)=\frac{1}{\sqrt{2 \pi}} \operatorname{dist}\left(t^{\bar{\lambda}-2 \sigma_{0}} \chi_{(0,1)}(t), K_{r}\right)=\frac{d_{r}(\lambda)}{\sqrt{2 \pi}} .
$$

We conclude the proof of Theorem 2.2 using $\left\|k_{\lambda}\right\|_{2}^{2}=\frac{1}{4 \pi\left(\Re(\lambda)-\sigma_{0}\right)}$.

Proof of Corollary 2.3. It follows from the proof of Theorem 2.2 and (5.4) that $L$ does not vanish on

$$
r-\sigma_{0}+\left\{\mu \in \mathbb{C}:\left|\frac{\mu-\lambda}{\mu+\bar{\lambda}-2 \sigma_{0}}\right|<\sqrt{4 \pi\left(\Re(\lambda)-\sigma_{0}\right)} \sup _{\substack{h \in E_{r} \\\|h\|_{2}=1}}|h(\lambda)|\right\} .
$$

So in particular (with $h=h_{A, r}$ ), we get that $L$ does not vanish on

$$
r-\sigma_{0}+\left\{\mu \in \mathbb{C}:\left|\frac{\mu-\lambda}{\mu+\bar{\lambda}-2 \sigma_{0}}\right|<\sqrt{4 \pi\left(\Re(\lambda)-\sigma_{0}\right)} \frac{\left|h_{A, r}(\lambda)\right|}{\left\|h_{A, r}\right\|_{2}}\right\},
$$

which proves Corollary 2.3 because $h_{A, r}=\mathcal{M} f_{A, r}=\frac{1}{\sqrt{2 \pi}} \widehat{f_{A, r}}$. 
The following could be interesting in applications.

Corollary 5.2. Let $M$ be a subspace of $K_{r}$ and $\lambda \in \Pi_{\sigma_{0}}$. Then $L$ does not vanish on the disc

$$
r-\sigma_{0}+\left\{\mu \in \mathbb{C}:\left|\frac{\mu-\lambda}{\mu+\bar{\lambda}-2 \sigma_{0}}\right|^{2}<1-2\left(\Re(\lambda)-\sigma_{0}\right) \operatorname{dist}^{2}\left(t^{\bar{\lambda}-2 \sigma_{0}} \chi_{(0,1)}, M\right)\right\} .
$$

Proof. It is sufficient to note that

$$
\operatorname{dist}\left(t^{\bar{\lambda}-2 \sigma_{0}} \chi_{(0,1)}, M\right) \geq \operatorname{dist}\left(t^{\bar{\lambda}-2 \sigma_{0}} \chi_{(0,1)}, K_{r}\right)=d_{r}(\lambda)
$$

and then apply Theorem 2.2

Adapting the proof of Theorem 2.2, we could immediately obtain the following generalization.

Theorem 5.3. Let $k \in \mathbb{N}$ and $\lambda \in \Pi_{\sigma_{0}}$. The function $L$ does not have any zero of order greater than or equal to $k$ on

$$
r-\sigma_{0}+\left\{\mu \in \mathbb{C}:\left|\frac{\mu-\lambda}{\mu+\bar{\lambda}-2 \sigma_{0}}\right|<\left(1-2\left(\Re(\lambda)-\sigma_{0}\right) d_{r}^{2}(\lambda)\right)^{\frac{1}{2 k}}\right\} .
$$

\section{A Beurling-Nyman type theorem For Dirichlet SERIES}

One of the main steps for proving Theorem 2.4 is to prove that the space $E_{r}$ is a closed subspace of $H^{2}\left(\Pi_{\sigma_{0}}\right)$ that is invariant under the multiplication operator $\tau_{v}$, $v \geq 0$, and then to apply Lax-Beurling's theorem. Before recalling this theorem, we give some notation and results. We refer to [Nik02, Part A, Chapters 2 \& 6] for more details.

For $v \in \mathbb{R}$, let $\tau_{v}$ be the operator of multiplication on $L^{2}\left(\sigma_{0}+i \mathbb{R}\right)$ defined by

$$
\left(\tau_{v} f\right)\left(\sigma_{0}+i t\right)=e^{-i v t} f\left(\sigma_{0}+i t\right) \quad\left(f \in L^{2}\left(\sigma_{0}+i \mathbb{R}\right)\right) .
$$

We also denote by $H^{\infty}\left(\Pi_{\sigma_{0}}\right)$ the Hardy space of bounded analytic functions on $\Pi_{\sigma_{0}}$; as in $H^{2}\left(\Pi_{\sigma_{0}}\right)$, functions in $H^{\infty}\left(\Pi_{\sigma_{0}}\right)$ admit "radial" limits at almost every point of the boundary $\Re(s)=\sigma_{0}$ and if $f \in H^{\infty}\left(\Pi_{\sigma_{0}}\right)$ and $f^{*}$ is its boundary limits, then $f^{*} \in L^{\infty}\left(\sigma_{0}+i \mathbb{R}\right)$ and $\left\|f^{*}\right\|_{\infty}=\|f\|_{\infty}\left(=\sup _{z \in \Pi_{\sigma_{0}}}|f(z)|\right)$. A function $\Theta$ in $H^{\infty}\left(\Pi_{\sigma_{0}}\right)$ is said to be inner if $\left|\Theta\left(\sigma_{0}+i t\right)\right|=1$ for almost every point $t \in \mathbb{R}$.

It is easy to see that if $\Theta$ is inner and $E=\Theta H^{2}\left(\Pi_{\sigma_{0}}\right)$, then $E$ is a closed subspace of $H^{2}\left(\Pi_{\sigma_{0}}\right)$ invariant by $\tau_{v}, v \geq 0$. Lax-Beurling's theorem gives the converse.

Theorem 6.1 (Lax-Beurling). Let $E$ be a closed subspace of $H^{2}\left(\Pi_{\sigma_{0}}\right)$ such that $\tau_{v} E \subset E, \forall v \geq 0$. Then there is an inner function $\Theta \in H^{\infty}\left(\Pi_{\sigma_{0}}\right)$ unique (up to a constant of modulus one) such that $E=\Theta H^{2}\left(\Pi_{\sigma_{0}}\right)$.

Remark 6.2. When $E$ is spanned by a family of functions, we can make a little bit more precise the conclusion of Theorem 6.1. Indeed, let $E$ be a closed subspace of $H^{2}\left(\Pi_{\sigma_{0}}\right)$ spanned by a family of functions $\left(f_{i}\right)_{i \in I}, f_{i} \in H^{2}\left(\Pi_{\sigma_{0}}\right)$ and let $f_{i}=h_{i} g_{i}$ be the factorization of $f_{i}$ into an inner factor $h_{i}$ and an outer factor $g_{i}$. If $\tau_{v} E \subset E$, $\forall v \geq 0$, then $E=\Theta H^{2}\left(\Pi_{\sigma_{0}}\right)$, where $\Theta=\operatorname{gcd}\left(h_{i}: i \in I\right)$ is the greatest common inner divisor of the family $\left(h_{i}\right)_{i \in I}$.

Proof of Theorem 2.4. First of all, note that

$$
\bigcap_{h \in E_{r}} h^{-1}(\{0\})=\left\{s \in \Pi_{\sigma_{0}}: L\left(s+r-\sigma_{0}\right)=0\right\},
$$


where we recall that

$$
E_{r}=\mathcal{M} K_{r}=\operatorname{span}_{H^{2}\left(\Pi_{\sigma_{0}}\right)}\left(h_{A, r}: A \text { an } m_{L} \text {-admissible sequence }\right)
$$

and

$$
h_{A, r}(s)=-\frac{1}{\sqrt{2 \pi}} L\left(s+r-\sigma_{0}\right) \hat{\varphi}\left(s+r-\sigma_{0}\right) g_{A}\left(s+r-\sigma_{0}\right), \quad \Re(s)>\sigma_{0} .
$$

Indeed, the first inclusion

$$
\left\{s \in \Pi_{\sigma_{0}}: L\left(s+r-\sigma_{0}\right)=0\right\} \subset \bigcap_{h \in E_{r}} h^{-1}(\{0\})
$$

is trivial. For the converse inclusion, remember that, by assumption, $\hat{\varphi}$ does not vanish on $\Pi_{r}$. Then it is sufficient to notice that, according to Lemma 4.2, the only common zero to the functions $g_{A}\left(s+r-\sigma_{0}\right)$ is $s=1-r+\sigma_{0}$, but this zero is compensated by the singularities of $L\left(s+r-\sigma_{0}\right)$ at this point.

The proofs of $(4) \Longrightarrow(3)$ and $(3) \Longrightarrow(2)$ are trivial.

$(2) \Longrightarrow(1)$ : Let $\lambda \in \Pi_{\sigma_{0}}$ such that $d_{r}(\lambda)=0$. Then according to Theorem 2.2 , the function $L$ does not vanish on

$$
r-\sigma_{0}+\left\{\mu \in \mathbb{C}:\left|\frac{\mu-\lambda}{\mu+\bar{\lambda}-2 \sigma_{0}}\right|<1\right\},
$$

and it follows from the paragraph after Theorem 2.2 that this region is precisely the half-plane $\Pi_{r}$.

$(1) \Longrightarrow(4)$ : Denote by $S_{\beta}$ the "shift" on $L_{*}^{2}\left((0,1), \frac{d t}{t^{1-2 \sigma_{0}}}\right)$ defined by

$$
\left(S_{\beta} f\right)(t):=\beta^{-\sigma_{0}} f\left(\frac{t}{\beta}\right), \quad 0<\beta \leq 1,0 \leq t \leq 1 .
$$

It is clear that $S_{\beta}$ is a unitary operator on $L_{*}^{2}\left((0,1), \frac{d t}{t^{1-2 \sigma_{0}}}\right)$. We will show that

$$
S_{\beta} K_{r} \subset K_{r} \quad(0<\beta \leq 1) .
$$

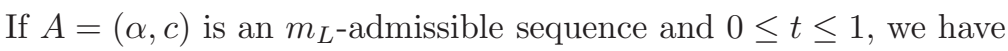

$$
\begin{aligned}
\left(S_{\beta} f_{A, r}\right)(t) & =\beta^{-\sigma_{0}} f_{A, r}\left(\frac{t}{\beta}\right) \\
& =\beta^{-\sigma_{0}} \frac{t^{r-\sigma_{0}}}{\beta^{r-\sigma_{0}}} \sum_{j=1}^{\ell(\alpha)} c_{j} \psi_{k}\left(\frac{\alpha_{j} \beta}{t}\right) \\
& =\beta^{-r} f_{A^{\prime}, r}(t),
\end{aligned}
$$

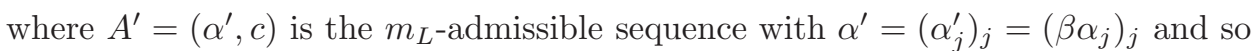
$f_{A^{\prime}, r} \in K_{r}$. Hence for every admissible sequence $A$, we have proved that $S_{\beta} f_{A, r} \in$ $K_{r}$. Since $S_{\beta}$ is a bounded operator and the functions $f_{A, r}$ span the subspace $K_{r}$, we deduce (6.2). Therefore we obtain that $E_{r}=\mathcal{M} K_{r}$ is a closed subspace of $H^{2}\left(\Pi_{\sigma_{0}}\right)$ which is invariant under the semi-group of operators $\mathcal{M} S_{\beta} \mathcal{M}^{-1}, 0<\beta \leq 1$. Now let us show that

$$
\mathcal{M} S_{\beta} \mathcal{M}^{-1}=\tau_{v}
$$


with $v=-\log \beta$. If $h \in H^{2}\left(\Pi_{\sigma_{0}}\right)$ and if $f \in L_{*}^{2}\left((0,1), \frac{d t}{t^{1-2 \sigma_{0}}}\right)$ is such that $\mathcal{M}^{-1} h=$ $f$, we have for $\Re(s)>\sigma_{0}$,

$$
\begin{aligned}
\left(\mathcal{M} S_{\beta} \mathcal{M}^{-1} h\right)(s) & =\frac{1}{\sqrt{2 \pi}} \int_{0}^{\infty}\left(S_{\beta} f\right)(t) t^{s-1} d t \\
& =\frac{1}{\sqrt{2 \pi}} \int_{0}^{+\infty} \beta^{-\sigma_{0}} f\left(\frac{t}{\beta}\right) t^{s-1} d t \\
& =\beta^{s-\sigma_{0}} \frac{1}{\sqrt{2 \pi}} \int_{0}^{+\infty} f(u) u^{s-1} d u \\
& =\beta^{s-\sigma_{0}}(\mathcal{M} f)(s)=\beta^{s-\sigma_{0}} h(s) .
\end{aligned}
$$

Hence we have

$$
\left(\mathcal{M} S_{\beta} \mathcal{M}^{-1} h\right)\left(\sigma_{0}+i t\right)=\beta^{i t} h\left(\sigma_{0}+i t\right)=e^{i t \log \beta} h\left(\sigma_{0}+i t\right)=\left(\tau_{v} h\right)\left(\sigma_{0}+i t\right),
$$

with $v=-\log \beta$, which proves (6.3). We therefore obtain that $\tau_{v} E_{r} \subset E_{r}$, for all $v \geq 0$ and then Lax-Beurling's theorem (see Theorem 6.1) implies that there is an inner function $\Theta$ in the half-plane $\Pi_{\sigma_{0}}$ such that $E_{r}=\Theta H^{2}\left(\Pi_{\sigma_{0}}\right)$. Moreover we know that $\Theta=B S$, where $B$ is a Blaschke product and $S$ is a singular inner function ([Nik02]). But according to Remark 6.2, the zeros of $B$ coincide with the set of common zeros of functions $h \in E_{r}$. Hence it follows, from (6.1) and the hypothesis, that $B$ has no zeros. In other words, $B \equiv 1$. We will now show that $S \equiv 1$. First note that since $h_{A, r}$ is analytic on $\Re(s)>2 \sigma_{0}-r$ and since $S$ is a common inner divisor of all functions in $E_{r}$, it follows that $S$ can be continued analytically through the axis $\sigma_{0}+i \mathbb{R}$. In particular, this forces $S$ to be of the form $S(s)=e^{-a\left(s-\sigma_{0}\right)}$, for some $a \geq 0$ (see for instance Nik02, Part A, Chapters $4 \&$ $6])$. Now let $h_{A, r}$ be a function in $E_{r}$ and write $h_{A, r}=S h$, with $h \in H^{2}\left(\Pi_{\sigma_{0}}\right)$.

Lemma 6.3. Let $h \in H^{2}\left(\Pi_{\sigma_{0}}\right)$. Then

$$
\limsup _{\sigma \rightarrow+\infty} \frac{\log |h(\sigma)|}{\sigma} \leq 0 .
$$

Proof of Lemma 6.3. Using (5.2) and (5.4), we get

$$
|h(\sigma)| \leq\|h\|_{2}\left\|k_{\sigma}\right\|_{2}=\frac{\|h\|_{2}}{2 \sqrt{\pi}\left(\sigma-\sigma_{0}\right)^{1 / 2}},
$$

for every $\sigma>\sigma_{0}$. Hence

$$
\frac{\log |h(\sigma)|}{\sigma} \leq \frac{O(1)}{\sigma}-\frac{1}{2} \frac{\log \left(\sigma-\sigma_{0}\right)}{\sigma},
$$

which gives the result letting $\sigma \rightarrow+\infty$.

On the one hand, according to the previous lemma, we have

$$
\limsup _{x \rightarrow+\infty} \frac{\log \left|h_{A, r}(x)\right|}{x} \leq \limsup _{x \rightarrow+\infty} \frac{\log |S(x)|}{x}=-a \leq 0 .
$$

On the other hand, writing

$$
h_{A, r}(x)=L\left(x+r-\sigma_{0}\right) \hat{\varphi}\left(x+r-\sigma_{0}\right) \sum_{j=1}^{\ell(\alpha)} c_{j} \alpha_{j}^{x+r-\sigma_{0}},
$$


we can assume, by Lemma 4.2, that $0<\alpha_{1}<\cdots<\alpha_{\ell(\alpha)}=1$ and that $c_{\ell(\alpha)} \neq 0$. Then we have

$$
\left|h_{A, r}(x)\right| \sim\left|a_{1}\right|\left|\hat{\varphi}\left(x+r-\sigma_{0}\right) c_{\ell(\alpha)}\right|, \quad x \rightarrow+\infty .
$$

Hence we can find $x_{0}>1$ such that

$$
\left|h_{A, r}(x)\right| \geq \frac{\left|a_{1}\right|}{2}\left|\hat{\varphi}\left(x+r-\sigma_{0}\right) c_{\ell(\alpha)}\right| \quad\left(x>x_{0}\right),
$$

which gives

$$
\log \left|h_{A, r}(x)\right| \geq-\log 2+\log \left|a_{1}\right|+\log \left|c_{\ell(\alpha)}\right|+\log \left|\hat{\varphi}\left(x+r-\sigma_{0}\right)\right|\left(x>x_{0}\right) .
$$

Therefore we obtain that

$$
\limsup _{x \rightarrow+\infty} \frac{\log \left|h_{A, r}(x)\right|}{x} \geq \limsup _{x \rightarrow+\infty} \frac{\log \left|\hat{\varphi}\left(x+r-\sigma_{0}\right)\right|}{x}=0 .
$$

Using (6.4), we conclude that $a=0$. Hence $S \equiv 1$, which gives $E_{r}=H^{2}\left(\Pi_{\sigma_{0}}\right)$, that is, $K_{r}=L_{*}^{2}\left((0,1), \frac{d t}{t^{1-2 \sigma_{0}}}\right)$.

Remark 6.4. In fact, with the hypothesis on $\varphi$, one can easily show that we always have $\lim \sup _{x \rightarrow+\infty} \frac{\log \left|\hat{\varphi}\left(x+r-\sigma_{0}\right)\right|}{x} \leq 0$. Note that there exists some $\varphi$ for which the limsup is negative.

\section{Some EXAmples And APPliCations}

7.1. The Riemann zeta function. Let

$$
\zeta(s)=\sum_{n \geq 1} \frac{1}{n^{s}} \quad(\Re(s)>1)
$$

be the Riemann zeta function. Then it is well known that $\zeta$ can be meromorphically continued in the whole plane $\mathbb{C}$, with a unique pole of order 1 at the point $s=1$ (Tit86]). Thus, the function $\zeta$ satisfies our hypothesis with $m_{L}=1$ (and for instance, $\left.\sigma_{0}=0\right)$. Now let us consider the function $\varphi$ defined on $[0,+\infty[$ by

$$
\varphi(t)= \begin{cases}(1-t)^{-\sigma_{1}} & \text { if } 0 \leq t<1 \\ 0 & \text { if } t \geq 1\end{cases}
$$

where $\sigma_{1}<1 / 2$ is fixed.

Then an elementary computation shows that $\hat{\varphi}(s)=\frac{\Gamma(s) \Gamma\left(1-\sigma_{1}\right)}{\Gamma\left(s+1-\sigma_{1}\right)}, \Re(s)>0$. Hence,

$$
|\hat{\varphi}(s)| \sim \frac{\Gamma\left(1-\sigma_{1}\right)}{|t|^{1-\sigma_{1}}} \quad \text { as }|t|=|\Im(s)| \rightarrow \infty .
$$

We now illustrate 1 the use of Theorem 2.1 in order to determine the values of $r$ such that

$$
\psi \in L^{2}\left((1, \infty), \frac{d u}{u^{1+2 r}}\right) .
$$

An obvious computation shows that

$$
\psi(u)=\frac{u}{1-\sigma_{1}}-\sum_{n<u} \frac{1}{\left(1-\frac{n}{u}\right)^{\sigma_{1}}}, \quad u>0 .
$$

\footnotetext{
${ }^{1}$ It is an illustration since here Theorem 2.1 is useless, as can be seen in Theorem 7.2
} 
Let $\mu=\mu(1 / 2)$ be a convexity bound for $\zeta(1 / 2+i t)$ (Ten08]), so that we have for all $\varepsilon>0$,

$$
|\zeta(r+i t)|= \begin{cases}O_{\varepsilon}\left(|t|^{\frac{1}{2}-(1-2 \mu) r+\varepsilon}\right) & \text { if } 0 \leq r \leq 1 / 2, \\ O_{\varepsilon}\left(|t|^{2 \mu(1-r)+\varepsilon}\right) & \text { if } 1 / 2 \leq r<1 .\end{cases}
$$

We get from (7.1),

$$
|\zeta(r+i t) \hat{\varphi}(r+i t)|^{2}= \begin{cases}O_{\varepsilon}\left(|t|^{-1-(2-4 \mu) r+2 \sigma_{1}+\varepsilon}\right) & \text { if } 0 \leq r \leq 1 / 2, \\ O_{\varepsilon}\left(|t|^{-2+2 \sigma_{1}+4 \mu(1-r)+\varepsilon}\right) & \text { if } 1 / 2 \leq r<1\end{cases}
$$

So a direct application of Theorem 2.1 gives:

Proposition 7.1. With the notation above, then $\psi \in L^{2}\left((1, \infty), \frac{d u}{u^{1+2 r}}\right)$ if one of the following holds:

- $\max \left(0, \sigma_{1} /(1-2 \mu)\right)<r \leq 1 / 2$;

- $\max \left(1 / 2,1-\left(1-2 \sigma_{1}\right) /(4 \mu)\right)<r<1$;

- $r>1$.

By a result of Huxley ([Hux05]), we can take $\mu=\frac{32}{205}$. In particular, one may have $r=1 / 2$ if $\sigma_{1}$ is chosen such that $\sigma_{1}<\frac{141}{410} \approx 0.343 \ldots$

If we assume the Lindelöf hypothesis, we have $\mu=0$, and a similar computation as above implies that if $r \neq 1$ and $r>\max \left(0, \sigma_{1}\right)$, then

$$
\psi \in L^{2}\left((1, \infty), \frac{d u}{u^{1+2 r}}\right) .
$$

In fact, the work above is useless for the Riemann zeta function since we can directly prove the following:

Theorem 7.2. Let $r \neq 1$. Then $\psi \in L^{2}\left((1, \infty), \frac{d u}{u^{1+2 r}}\right)$ if and only if $r>\max \left(0, \sigma_{1}\right)$. Moreover, if the condition holds we have

$$
\|\psi\|_{L^{2}\left((1, \infty), \frac{d u}{u^{1+2 r}}\right)}^{2} \leq \frac{1}{2 r}+C\left(\sigma_{1}\right) \zeta\left(1+2 r-2 \sigma_{1}\right)
$$

where

$$
C\left(\sigma_{1}\right)=\frac{1}{1-2 \sigma_{1}}+\frac{1}{\left(1-\sigma_{1}\right)^{2}\left(3-2 \sigma_{1}\right)}+\frac{\varepsilon_{1}}{\left(1-\sigma_{1}\right)^{2}}
$$

and $\varepsilon_{1}=1$ if $\sigma_{1} \geq 0$ and $\varepsilon_{1}=-1$ if $\sigma_{1}<0$.

Proof. In the case of the zeta function and for the previous choice of $\varphi$, we have

$$
\psi(u)=\frac{u}{1-\sigma_{1}}-\sum_{n<u}\left(1-\frac{n}{u}\right)^{-\sigma_{1}} \quad(u>0) .
$$

Hence

$$
\begin{aligned}
\|\psi\|_{2}^{2} & =\int_{1}^{\infty}\left|\frac{u}{1-\sigma_{1}}-\sum_{n<u}\left(1-\frac{n}{u}\right)^{-\sigma_{1}}\right|^{2} \frac{d u}{u^{2 r+1}} \\
& =\int_{1}^{\infty}\left|\frac{u^{1-\sigma_{1}}}{1-\sigma_{1}}-\sum_{n<u}(u-n)^{-\sigma_{1}}\right|^{2} \frac{d u}{u^{1+2 r-2 \sigma_{1}}} \\
& =\sum_{k=1}^{\infty} \int_{k}^{k+1}\left|\Delta_{k}(u)\right|^{2} \frac{d u}{u^{1+2 r-2 \sigma_{1}}},
\end{aligned}
$$


where

$$
\Delta_{\ell}(u)=\frac{u^{1-\sigma_{1}}}{1-\sigma_{1}}-\sum_{n=1}^{\ell}(u-n)^{-\sigma_{1}} \quad(1 \leq \ell<u) .
$$

For $1 \leq \ell<u$, we will now prove that

$$
\left|\Delta_{\ell}(u)\right| \leq\left|u^{-\sigma_{1}}-(u-\ell)^{-\sigma_{1}}\right|+\frac{(u-\ell)^{1-\sigma_{1}}}{1-\sigma_{1}} .
$$

With the definition of $\varepsilon_{1}$, the function $t \mapsto \varepsilon_{1}(u-t)^{-\sigma_{1}}$ is increasing for $t \in[0, u)$. Then

$\varepsilon_{1} u^{-\sigma_{1}}+\varepsilon_{1} \int_{0}^{\ell}(u-t)^{-\sigma_{1}} d t \leq \varepsilon_{1} \sum_{n=0}^{\ell}(u-n)^{-\sigma_{1}} \leq \varepsilon_{1}(u-\ell)^{-\sigma_{1}}+\varepsilon_{1} \int_{0}^{\ell}(u-t)^{-\sigma_{1}} d t$,

whence

$$
\varepsilon_{1} u^{-\sigma_{1}}-\varepsilon_{1}(u-\ell)^{-\sigma_{1}}+\varepsilon_{1} \frac{(u-\ell)^{1-\sigma_{1}}}{1-\sigma_{1}} \leq \varepsilon_{1} \Delta_{\ell}(u) \leq \varepsilon_{1} \frac{(u-\ell)^{1-\sigma_{1}}}{1-\sigma_{1}},
$$

which immediately gives (7.2). Applying (7.2) gives

$$
\|\psi\|_{2}^{2} \leq \sum_{k=1}^{\infty} \int_{0}^{1}\left(\left|(u+k)^{-\sigma_{1}}-u^{-\sigma_{1}}\right|+\frac{u^{1-\sigma_{1}}}{1-\sigma_{1}}\right)^{2} \frac{d u}{(u+k)^{1+2 r-2 \sigma_{1}}} .
$$

Using the fact that $\left|(u+k)^{-\sigma_{1}}-u^{-\sigma_{1}}\right|=\varepsilon_{1}\left(u^{-\sigma_{1}}-(u+k)^{-\sigma_{1}}\right)$ and expanding each integral, we get

$$
\|\psi\|_{2}^{2} \leq \int_{0}^{1}\left(\theta_{2 \sigma_{1}}(u) \xi_{\sigma_{1}}(u)^{2}-2 \theta_{\sigma_{1}}(u) \xi_{\sigma_{1}}(u)+\theta_{0}(u)\right) d u
$$

where we set

$$
\xi_{\sigma_{1}}(u)=u^{-\sigma_{1}}+\varepsilon_{1} \frac{u^{1-\sigma_{1}}}{1-\sigma_{1}}
$$

and

$$
\theta_{A}(u)=\sum_{k \geq 1} \frac{1}{(u+k)^{1+2 r-A}} \quad(A<2 r)
$$

It is now clear that

$$
\theta_{\sigma_{1}}(u) \xi_{\sigma_{1}}(u) \geq 0 \quad\left(0 \leq u \leq 1, \sigma_{1}<1 / 2\right)
$$

(note that by assumption $\sigma_{1}<1 / 2$ ). Hence

$$
\|\psi\|_{2}^{2} \leq \int_{0}^{1}\left(\theta_{2 \sigma_{1}}(u) \xi_{\sigma_{1}}(u)^{2}+\theta_{0}(u)\right) d u .
$$

A simple computation yields $\int_{0}^{1} \theta_{0}(u) d u=\frac{1}{2 r}$. For the remaining term, noting that for any $A<2 r$, we have $\theta_{A}(u) \leq \zeta(1+2 r-A)$, we obtain

$$
\|\psi\|_{2}^{2} \leq \frac{1}{2 r}+\zeta\left(1+2 r-2 \sigma_{1}\right) \int_{0}^{1} \xi_{\sigma_{1}}(u)^{2} d u
$$

which gives the first part of the theorem after some obvious computations.

On the other hand, suppose that $\sigma_{1}>0$. (If $\sigma_{1} \leq 0$ there is nothing to prove since by hypothesis $r>\sigma_{0}$ and $\sigma_{0}=0$ in our case.) 
It suffices to find $U\left(\sigma_{1}\right)>0$ such that

$$
\int_{k}^{k+1}\left|\Delta_{k}(u)\right|^{2} d u \geq U\left(\sigma_{1}\right)
$$

uniformly in $k$. In that case, we shall have

$$
\begin{aligned}
\|\psi\|_{2}^{2} & =\sum_{k=1}^{\infty} \int_{k}^{k+1}\left|\Delta_{k}(u)\right|^{2} \frac{d u}{u^{1+2 r-2 \sigma_{1}}} \\
& \geq \sum_{k=1}^{\infty} \frac{1}{(k+1)^{1+2 r-2 \sigma_{1}}} \int_{k}^{k+1}\left|\Delta_{k}(u)\right|^{2} d u \\
& \geq U\left(\sigma_{1}\right) \sum_{k=1}^{\infty} \frac{1}{(k+1)^{1+2 r-2 \sigma_{1}}}
\end{aligned}
$$

hence $r>\sigma_{1}$.

It now remains to prove (7.5). First by (7.2), for any $k<u \leq k+1$, we have

$$
\left|\Delta_{k-1}(u)\right| \leq\left|u^{-\sigma_{1}}-(u+1-k)^{-\sigma_{1}}\right|+\frac{(u+1-k)^{1-\sigma_{1}}}{1-\sigma_{1}} \leq 2+\frac{2^{1-\sigma_{1}}}{1-\sigma_{1}} .
$$

Let $\delta>0$ be fixed such that $\delta^{-\sigma_{1}}=2\left(2+\frac{2^{1-\sigma_{1}}}{1-\sigma_{1}}\right)$. Then $\delta<1$, and for such a choice of $\delta$, we have

$$
\left|\Delta_{k}(u)\right|=\left|(u-k)^{-\sigma_{1}}-\Delta_{k-1}(u)\right| \geq \frac{1}{2}(u-k)^{-\sigma_{1}},
$$

for any $k \geq 1$ and any $k<u<k+\delta$. Hence,

$$
\int_{k}^{k+1}\left|\Delta_{k}(u)\right|^{2} d u \geq \frac{1}{4} \int_{k}^{k+\delta}(u-k)^{-2 \sigma_{1}} d u=\frac{\delta^{1-2 \sigma_{1}}}{4\left(1-2 \sigma_{1}\right)}>0 .
$$

7.1.1. Zero-free discs for $\zeta$. Let $r>\max \left(0, \sigma_{1}\right), r \neq 1$. Then according to Theorem 7.2. the function $\psi \in L^{2}\left((1, \infty), \frac{d u}{u^{1+2 r}}\right)$. Take $\lambda \in \mathbb{C}, \Re(\lambda)>0$. It follows from Theorem 2.2 that the Riemann zeta function $s \longmapsto \zeta(s)$ does not vanish in the disc

$$
r+\left\{\mu \in \mathbb{C}:\left|\frac{\mu-\lambda}{\mu+\bar{\lambda}}\right|<\sqrt{1-2 \Re(\lambda) d_{r}^{2}(\lambda)}\right\},
$$

where $d_{r}(\lambda)=\operatorname{dist}\left(t^{\bar{\lambda}} \chi_{(0,1)}, K_{r}\right)$, with

$$
K_{r}=\operatorname{span}\left(f_{A, r}: A \text { a } 1 \text {-admissible sequence }\right) .
$$

As mentioned in Remark 4.4, with the choice $\varphi(t)=\chi_{(0,1)}(t)$, we recover Nik95, Theorem 0.1].

We will now try to give more explicit zero-free discs for the Riemann zeta function. So, let $A=(\alpha, c)$ be a 1-admissible sequence, which means that

$$
\sum_{j=1}^{\ell(\alpha)} c_{j} \alpha_{j}=0,
$$

with $0<\alpha_{j} \leq 1, c_{j} \in \mathbb{C}$. 
Applying Corollary 2.3 gives that $s \mapsto \zeta(s)$ does not vanish in the disc

$$
r+\left\{\mu \in \mathbb{C}:\left|\frac{\mu-\lambda}{\mu+\bar{\lambda}}\right|<\sqrt{2 \Re(\lambda)} \frac{\left|\zeta(\lambda+r) \hat{\varphi}(\lambda+r) \sum_{j=1}^{\ell(\alpha)} c_{j} \alpha_{j}^{\lambda+r}\right|}{\left\|f_{A, r}\right\|_{L_{*}^{2}((0,1), d t / t)}}\right\} .
$$

But the estimate (4.4) gives for $0<r<1$ :

$$
\left\|f_{A, r}\right\|_{L_{*}^{2}((0,1), d t / t)} \leq \sum_{j=1}^{\ell(\alpha)}\left|c_{j} \alpha_{j}^{r}\right|\left(\frac{1}{\left(1-\sigma_{1}\right) \sqrt{2-2 r}}+\|\psi\|_{L^{2}\left((1, \infty), \frac{d u}{u^{1+2 r}}\right)}\right)
$$

and thus we deduce

Proposition 7.3. Let $\max \left(0, \sigma_{1}\right)<r<1$ and let $A=(\alpha, c)$ be a 1-admissible sequence. If $\Re(\lambda)>0$, then $s \mapsto \zeta(s)$ does not vanish in the disc

$$
r+\left\{\mu \in \mathbb{C}:\left|\frac{\mu-\lambda}{\mu+\bar{\lambda}}\right|<\sqrt{2 \Re(\lambda)} \frac{\left|\left(\sum_{j=1}^{\ell(\alpha)} c_{j} \alpha_{j}^{\lambda+r}\right) \hat{\varphi}(\lambda+r) \zeta(\lambda+r)\right|}{\sum_{j=1}^{\ell(\alpha)}\left|c_{j} \alpha_{j}^{r}\right|\left(\|\psi\|_{2}+\frac{1}{\left(1-\sigma_{1}\right) \sqrt{2-2 r}}\right)}\right\} .
$$

In particular, if we consider only admissible sequences of length 2 , we obtain zero-free discs of the form

$$
r+\left\{\mu \in \mathbb{C}:\left|\frac{\mu-\lambda}{\mu+\bar{\lambda}}\right|<\sqrt{2 \Re(\lambda)} \frac{\left|\left(\alpha^{\lambda+r}-\alpha\right) \hat{\varphi}(\lambda+r) \zeta(\lambda+r)\right|}{\left(\alpha^{r}+\alpha\right)\left(\|\psi\|_{2}+\frac{1}{\left(1-\sigma_{1}\right) \sqrt{2-2 r}}\right)}\right\} .
$$

Since $|\hat{\varphi}(\lambda+r)|=O\left(1 /|\Im(\lambda)|^{1-\sigma_{1}}\right)$ we obtain a larger disc than in Nik95 whenever $\Im(\lambda)$ is large enough and $\sigma_{1}>0$. We emphasize the fact that our zero-free discs are explicit.

For example, if we take $\alpha=1 / 4$ in (7.6), we obtain the following zero-free region for zeta which can be improved but has the merit to be quite explicit:

Corollary 7.4. Let $r>\max \left(0, \sigma_{1}\right)$ and let $\lambda \in \mathbb{C}$ such that $\Re(\lambda)>0$. Then the Riemann zeta function does not vanish in the disc

$$
r+\left\{\mu \in \mathbb{C}:\left|\frac{\mu-\lambda}{\mu+\bar{\lambda}}\right|<F\left(\lambda, r, \sigma_{1}\right)\right\},
$$

where

$$
F\left(\lambda, r, \sigma_{1}\right)=\frac{\sqrt{2 \Re(\lambda)}\left|\left(\left(\frac{1}{4}\right)^{\lambda+r}-\frac{1}{4}\right) \Gamma(\lambda+r) \Gamma\left(1-\sigma_{1}\right) \zeta(\lambda+r)\right|}{\left(\left(\frac{1}{4}\right)^{r}+\frac{1}{4}\right)\left(C\left(r, \sigma_{1}\right)+\frac{1}{\left(1-\sigma_{1}\right) \sqrt{2-2 r}}\right)\left|\Gamma\left(\lambda+r+1-\sigma_{1}\right)\right|}
$$

with $C\left(r, \sigma_{1}\right)=\sqrt{1 / 2 r+C\left(\sigma_{1}\right) \zeta\left(1+2 r-2 \sigma_{1}\right)}$ (recall that $C\left(\sigma_{1}\right)$ is defined in Theorem 7.2 .

Recall that the discs in Corollary 7.4 are Euclidean discs of center $(x, y)$ and radius $R$, where:

$$
\begin{aligned}
x & =r+\Re(\lambda) \frac{1+F\left(\lambda, r, \sigma_{1}\right)^{2}}{1-F\left(\lambda, r, \sigma_{1}\right)^{2}}, \quad y=\Im(\lambda), \\
R & =\frac{2 \Re(\lambda) F\left(\lambda, r, \sigma_{1}\right)}{1-F\left(\lambda, r, \sigma_{1}\right)^{2}} .
\end{aligned}
$$

Taking, for example, $\lambda=0.01+50 i, r=0.49$ and $\sigma_{1}=0.4$, then a simple evaluation of $F\left(\lambda, r, \sigma_{1}\right)$ implies that $\zeta$ has no zero in the disc of center $\frac{1}{2}+50 i$ and radius $5.13 \times 10^{-6}$. Note that $\zeta$ has a zero at $s=\frac{1}{2}+49.773 \ldots i$ and at $s=\frac{1}{2}+52.970 \ldots i$. 
7.2. Dirichlet $L$-functions. Let $\chi$ be a nontrivial Dirichlet character of conductor $\operatorname{cond}(\chi)=q$ and let $L(\chi, s)=\sum_{n>1} \chi(n) n^{-s}$ be the (degree 1) associated Dirichlet function. This function has an analytic continuation to the whole complex plane. For simplicity, we take $\sigma_{1}=\sigma_{0}=0$ and

$$
\varphi(t)= \begin{cases}1 & \text { if } 0 \leq t<1 \\ 0 & \text { if } t \geq 1\end{cases}
$$

So we have $\psi(u)=-\sum_{n<u} \chi(n)$. Since $\psi$ is bounded, it belongs to $L^{2}((1, \infty)$, $\left.\frac{d u}{u^{1+2 r}}\right)$ for all $r>0$. The admissibility condition for the sequence $A$ is empty, and we may take

$$
f_{A, r}(t)=t^{r} \sum_{j=1}^{\ell} c_{j} \sum_{n<\alpha_{j} / t} \chi(n)
$$

for all $A=(\alpha, c)$, where $\ell \geq 0, \alpha \in(0,1]^{\ell}$ and $c \in \mathbb{C}^{\ell}$. Let $r$ be such that $1 / 2 \leq r<1$ and $\lambda=1-r$. For simplicity, we write $d_{r}=d_{r}(\lambda)$ so that

$$
d_{r}^{2}=\min _{c_{j}, \alpha_{j}}\left(\int_{0}^{1}\left|t^{1-r}-t^{r} \sum_{j=1}^{\ell} c_{j} \sum_{n<\alpha_{j} / t} \chi(n)\right|^{2} \frac{d t}{t}\right) .
$$

Furthermore, we will write $d_{r, \chi}$ if we need to specify the dependance on $\chi$. Observe that we have trivially $d_{r}^{2} \leq 1 /(2-2 r)$ (just take all the $c_{j}$ to be 0$)$. In fact, we can be a little bit more precise.

Proposition 7.5. We have $d_{r}^{2}<1 /(2-2 r)$.

Proof. Let $c \in \mathbb{C}$ and $0<\alpha \leq 1$. A short calculation gives

$$
\begin{aligned}
d_{r}^{2} \leq \int_{0}^{1} \mid t^{1-r}- & \left.c t^{r} \sum_{n<\alpha / t} \chi(n)\right|^{2} \frac{d t}{t} \\
& =\frac{1}{2-2 r}-2 \alpha \Re(c L(\chi, 1))+|c|^{2} \alpha^{2 r+1} \int_{1}^{\infty}\left|\sum_{n<u} \chi(n)\right|^{2} \frac{d u}{u^{1+2 r}}
\end{aligned}
$$

Since we know that $L(\chi, 1) \neq 1$ (see for example [IK04, p. 37]), one can choose a suitable $c$ such that the right-hand side of the equation above is less than $1 /(2-2 r)$.

Theorem 2.2 asserts that $L(\chi, s)$ does not vanish on the disc

$$
r+\left\{\mu \in \mathbb{C}:\left|\frac{\mu+r-1}{\mu-r+1}\right|<\sqrt{1-2(1-r) d_{r}^{2}}\right\} .
$$

Note that the proposition above implies that the disc is not empty. We deduce, in particular, that $L(\chi, \sigma)$ does not vanish on the real interval

$$
\sigma>1-\frac{\sqrt{1-2(1-r) d_{r}^{2}}-\left(1-2(1-r) d_{r}^{2}\right)}{d_{r}^{2}} .
$$


(We can easily check that the disc (7.7) contains $\sigma=1$ and it is known that $L(\chi, \sigma) \neq 0$ for $\sigma \geq 1$.) Hence, $L(\chi, \sigma)$ does not vanish on

$$
\sigma>1-(1-r) \sqrt{1-2(1-r) d_{r}^{2}}
$$

We expect (by the Riemann Hypothesis for Dirichlet functions) that $d_{r}=0$, which would imply that $L(\chi, s)$ does not vanish on $\Pi_{r}$. If we had "only"

$$
d_{r}^{2} \leq \frac{1}{2-2 r}-\frac{C^{2}}{2(\log q)^{2}(1-r)^{3}}
$$

for some constant $C>0$, it would imply (using (7.8) ) that $L(\chi, \sigma)$ does not vanish in the real interval $\sigma>1-C / \log q$. That proves Theorem 2.5 and we also immediately get he following.

Theorem 7.6. If there exists an absolute constant $C$ such that for every (real) character $\chi$ there exists $r \in[1 / 2,1)$ with

$$
d_{r, \chi}^{2}<\frac{1}{2-2 r}-\frac{C^{2}}{2(\log \operatorname{cond}(\chi))^{2}(1-r)^{3}},
$$

then there is no Siegel's zero for Dirichlet $L$-functions.

The previous theorem can be seen as a Beurling-Nyman criterion for Siegel's conjecture.

7.2.1. Explicit zero-free discs for $L(\chi, s)$. We now apply Corollary 2.3. Note that for the special choice of $\varphi$ we have made $\left(\varphi=\chi_{(0,1)}\right)$, we have $\hat{\varphi}(s)=1 / s$. Moreover, since the function $\psi$ is bounded, say $\|\psi\| \leq B$, we have

$$
\|\psi\|_{L^{2}\left((1, \infty), d u / u^{1+2 r}\right)} \leq B / \sqrt{2 r} .
$$

Then Corollary 2.3 asserts that $s \mapsto L(\chi, s)$ does not vanish in the disc

$$
r+\left\{\mu \in \mathbb{C}:\left|\frac{\mu-\lambda}{\mu+\bar{\lambda}}\right|<\sqrt{2 \Re(\lambda)} \frac{|L(\chi, \lambda+r)|}{|\lambda+r|} \frac{\sqrt{2 r}}{B}\right\} .
$$

Let us observe that the Pólya-Vinogradov theorem implies that $B \leq 2 \sqrt{q} \log q$, where $q=\operatorname{cond}(\chi)$ is the conductor of $\chi$. Note also that there are some improvements of the Pólya-Vinogradov inequality for some characters (see for example GS07]).

7.3. The Selberg class. Let $L(s)=\sum_{n \geq 1} a_{n} n^{-s}$ be an $L$-function in the Selberg class $\mathcal{S}(\mathrm{dR} 07 \mathrm{a}])$. We denote by $d$ its degree and by $m_{L}$ the order of its pole at $s=1$.

As for the Riemann zeta function, we take

$$
\varphi(t)= \begin{cases}(1-t)^{-\sigma_{1}} & \text { if } 0 \leq t<1 \\ 0 & \text { if } t \geq 1\end{cases}
$$

where $\sigma_{1}<1 / 2$. By the Phragmen-Lindelöf principle, we have that $L(r+i t)=$ $O_{\varepsilon}\left(t^{\frac{d}{2}(1-r)+\varepsilon}\right)$ for $0<r \leq 1 / 2$. Then we deduce from Theorem 2.1 and (7.1) that

$$
\psi \in L^{2}\left((1, \infty), \frac{d u}{u^{1+2 r}}\right)
$$


if the following inequality holds:

$$
\sigma_{1}<\frac{1}{2}-\frac{1-r}{2} d
$$

In particular, for $r=1 / 2$, this gives $\sigma_{1}<1 / 2-d / 4$, which is better than $\sigma_{1}<-d / 4$ obtained in dR07a. Nevertheless, we should mention that for $d<4$, we can in fact take $\sigma_{1}=0$ (see dR07b).

\section{ACKNOWLEDGMENTS}

We thank Anne de Roton for helpful discussions, and Michel Balazard and Emmanuel Kowalski for their suggestions. We would also like to thank the referee for his careful reading and valuable suggestions.

\section{REFERENCES}

[BD03] L. Báez-Duarte. A strengthening of the Nyman-Beurling criterion for the Riemann hypothesis. Atti Accad. Naz. Lincei Cl. Sci. Fis. Mat. Natur. Rend. Lincei (9) Mat. Appl., 14(1):5-11, 2003. MR2057270 (2005b:11135)

[BDBLS00] L. Báez-Duarte, M. Balazard, B. Landreau, and E. Saias. Notes sur la fonction $\zeta$ de Riemann. III. Adv. Math., 149(1):130-144, 2000. MR1742356 (2001j:11084)

[Beu55] A. Beurling. A closure problem related to the Riemann zeta-function. Proc. Nat. Acad. USA, 41(5):312-314, 1955. MR0070655(17:15a)

[BF84] H. Bercovici and C. Foias. A real variable restatement of Riemann's hypothesis. Israel J. Math., 48(1):57-68, 1984. MR768266 (86d:11065)

[BS98] M. Balazard and E. Saias. Notes sur la fonction $\zeta$ de Riemann. 1. Adv. Math., 139(2):310-321, 1998. MR 1654169 (99k:11133)

[Bur02] J.-F. Burnol. A lower bound in an approximation problem involving the zeros of the Riemann zeta function. Adv. Math., 170(1):56-70, 2002. MR.1929303(2003h:11107)

[dR06] A. de Roton. Une approche hilbertienne de l'hypothèse de Riemann généralisée. Bull. Soc. Math. France, 134(3):417-445, 2006. MR2246000 (2007h:11102)

[dR07a] A. de Roton. Généralisation du critère de Beurling-Nyman pour l'hypothèse de Riemann. Trans. Amer. Math. Soc., 359(12):6111-6126 (electronic), 2007. MR2336318 (2008i:11112)

[dR07b] A. de Roton. On the mean square of the error term for an extended Selberg class. Acta Arith., 126(1):27-55, 2007. MR2284311(2007j:11121)

[GS07] Andrew Granville and K. Soundararajan. Large character sums: pretentious characters and the Pólya-Vinogradov theorem. J. Amer. Math. Soc., 20(2):357-384 (electronic), 2007. MR 2276774(2007k:11128)

[HIP27] G. Hardy, A. Ingham, and G. Pólya. Theorems concerning mean values of analytic functions. Proc. of the Royal Math. Soc., 113:542-569, 1927.

[Hof62] K. Hoffman. Banach spaces of analytic functions. Prentice-Hall Series in Modern Analysis. Prentice-Hall Inc., Englewood Cliffs, NJ, 1962. MR0133008 (24:A2844)

[Hux05] M. N. Huxley. Exponential sums and the Riemann zeta function. V. Proc. London Math. Soc. (3), 90(1):1-41, 2005. MR2107036 (2005h:11180)

[IK04] H. Iwaniec and E. Kowalski. Analytic number theory, volume 53 of American Mathematical Society Colloquium Publications. American Mathematical Society, Providence, RI, 2004. MR2061214 (2005h:11005)

[Nik95] N. Nikolski. Distance formulae and invariant subspaces, with an application to localization of zeros of the Riemann $\zeta$-function. Ann. Inst. Fourier (Grenoble), 45(1):143159, 1995. MR 1324128(96c:47005)

[Nik02] N. Nikolski. Operators, functions, and systems: an easy reading. Vol. 1, Hardy, Hankel, and Toeplitz, volume 92 of Mathematical Surveys and Monographs. American Mathematical Society, Providence, RI, 2002. Translated from the French by Andreas Hartmann. MR1864396 (2003i:47001a)

[Nym50] B. Nyman. On some groups and semi-groups of translations. Ph.D, thesis, Upsala, 1950 . 
[Ten08] G. Tenenbaum. Introduction à la théorie analytique et probabiliste des nombres. 3ème édition. Belin, 2008.

[Tit86] E. C. Titchmarsh. The theory of the Riemann zeta-function. The Clarendon Press, Oxford University Press, New York, second edition, 1986. Edited and with a preface by D. R. Heath-Brown. MR882550(88c:11049)

Université de Franche-Comté, Laboratoire de Mathématiques de Besançon, CNRS UMR 6623, 16, route de Gray, F-25030 Besançon, France

E-mail address: christophe.delaunay@univ-fcomte.fr

Laboratoire Paul Painlevé, UMR no. 8524, BÂt. M2, Université de Lille 1, 59655 Villeneuve D'Ascq Cedex, France

E-mail address: fricain@math.univ-lille1.fr

Université de Lyon, Université Lyon 1, Institut Camille Jordan CNRS UMR 5208, 43, Boulevard du 11 Novembre 1918, F-69622 Villeurbanne, France

E-mail address: mosaki@math.univ-lyon1.fr

Université de Lyon, Université de Saint-Etienne, LamUSE (EA 3989), 23, RUe du Dr P. Michelon, F-42000, SAint-Etienne, France

E-mail address: olivier.robert@univ-st-etienne.fr 